\title{
Implications of the Warm Corona and Relativistic Reflection Models for the Soft Excess in Mrk 509
}

\author{
Javier A. García ${ }^{1,2}$ (D) , Erin Kara ${ }^{3}$ (D), Dominic Walton ${ }^{4}$ (D), Tobias Beuchert ${ }^{5}$, Thomas Dauser ${ }^{2}$ (D), Efrain Gatuzz ${ }^{6}$ (D), \\ Mislav Balokovic ${ }^{7}$ (D), James F. Steiner ${ }^{8}$ (D) , Francesco Tombesi $^{3,9,10,11}$ (D), Riley M. T. Connors ${ }^{1}$ (D), Timothy R. Kallman ${ }^{11}$ (D), \\ Fiona A. Harrison ${ }^{1}$ (D) , Andrew Fabian ${ }^{4}$ (D) , Jörn Wilms ${ }^{2}$ (D), Daniel Stern ${ }^{12}$ (D), Lauranne Lanz ${ }^{13}$ (D), Claudio Ricci ${ }^{14,15,16}$ (D), and \\ David R. Ballantyne ${ }^{17}$ (iD \\ ${ }^{1}$ Cahill Center for Astronomy and Astrophysics, California Institute of Technology, Pasadena, CA 91125, USA; javier@ caltech.edu \\ ${ }^{2}$ Dr. Karl Remeis-Observatory and Erlangen Centre for Astroparticle Physics, Sternwartstr. 7, D-96049 Bamberg, Germany \\ ${ }^{3}$ Department of Astronomy, University of Maryland, College Park, MD 20742, USA \\ ${ }^{4}$ Institute of Astronomy, Madingley Road, Cambridge CB3 OHA, UK \\ ${ }^{5}$ Anton Pannekoek Institute for Astronomy, Universiteit van Amsterdam, Science Park 904, 1098 XH, Amsterdam, The Netherlands \\ ${ }^{6}$ ESO, Karl-Schwarzschild-Strasse 2, D-85748 Garching bei München, Germany \\ ${ }^{7}$ Harvard-Smithsonian Center for Astrophysics, 60 Garden Street, Cambridge, MA 02140, USA \\ ${ }_{9}^{8}$ MIT Kavli Institute for Astrophysics and Space Research, MIT, 70 Vassar Street, Cambridge, MA 02139, USA \\ ${ }^{9}$ Dipartimento di Fisica, Università di Roma "Tor Vergata", via della Ricerca Scientifica 1, I-00133, Roma, Italy \\ ${ }^{10}$ INAF Astronomical Observatory of Rome, Via Frascati 33, I-00078 Monteporzio Catone, Italy \\ ${ }^{11}$ NASA/Goddard Space Flight Center, Code 662, Greenbelt, MD 20771, USA \\ 12 Jet Propulsion Laboratory, California Institute of Technology, 4800 Oak Grove Drive, Mail Stop 169-221, Pasadena, CA 91109, USA \\ 13 Department of Physics and Astronomy, Dartmouth College, 6127 Wilder Laboratory, Hanover, NH 03755, USA
${ }^{14}$ Núcleo de Astronomía de la Facultad de Ingeniería, Universidad Diego Portales, Av. Ejército Libertador 441, Santiago, Chile \\ ${ }^{15}$ Kavli Institute for Astronomy and Astrophysics, Peking University, Beijing 100871, People's Republic of China \\ ${ }^{16}$ Chinese Academy of Sciences South America Center for Astronomy, Camino El Observatorio 1515, Las Condes, Santiago, Chile \\ ${ }^{17}$ Center for Relativistic Astrophysics, School of Physics, Georgia Institute of Technology, 837 State Street, Atlanta, GA 30332-0430, USA \\ Received 2018 September 24; revised 2018 November 26; accepted 2018 December 7; published 2019 January 24
}

\begin{abstract}
We present the analysis of the first Nuclear Spectroscopic Telescope Array observations ( $220 \mathrm{ks})$, simultaneous with the last Suzaku observations ( $\sim 50 \mathrm{ks})$, of the active galactic nucleus of the bright Seyfert 1 galaxy Mrk 509 . The time-averaged spectrum in the $1-79 \mathrm{keV} X$-ray band is dominated by a power-law continuum $(\Gamma \sim 1.8-1.9)$, a strong soft excess around $1 \mathrm{keV}$, and signatures of X-ray reflection in the form of $\mathrm{Fe} \mathrm{K}$ emission $(\sim 6.4 \mathrm{keV})$, an Fe $\mathrm{K}$ absorption edge $(\sim 7.1 \mathrm{keV})$, and a Compton hump due to electron scattering $(\sim 20-30 \mathrm{keV})$. We show that these data can be described by two very different prescriptions for the soft excess: a warm $(k T \sim 0.5-1 \mathrm{keV})$ and optically thick $(\tau \sim 10-20)$ Comptonizing corona or a relativistically blurred ionized reflection spectrum from the inner regions of the accretion disk. While these two scenarios cannot be distinguished based on their fit statistics, we argue that the parameters required by the warm corona model are physically incompatible with the conditions of standard coronae. Detailed photoionization calculations show that even in the most favorable conditions, the warm corona should produce strong absorption in the observed spectrum. On the other hand, while the relativistic reflection model provides a satisfactory description of the data, it also requires extreme parameters, such as maximum black hole spin, a very low and compact hot corona, and a very high density for the inner accretion disk. Deeper observations of this source are thus necessary to confirm the presence of relativistic reflection and further understand the nature of its soft excess.
\end{abstract}

Key words: accretion, accretion disks - black hole physics - galaxies: active - galaxies: individual (Mrk 509) galaxies: nuclei

\section{Introduction}

Accretion onto supermassive black holes in active galactic nuclei (AGNs) is one of the most efficient mechanisms to convert gravitational energy into radiation, comprised mostly of very energetic photons. For this reason, X-ray spectroscopy is a resourceful technique to study supermassive black holes and their interaction with their surroundings. In the case of most Seyfert AGNs, the X-ray continuum is typically dominated by a power law that extends to high energies, which is thought to be produced either in a central hot corona (e.g., Shakura \& Sunyaev 1973; Haardt 1993) or at the base of a jet (e.g., Matt et al. 1992; Markoff et al. 2005), although the exact mechanism is still a matter of study. Thermal emission from the accretion disk peaks in the ultraviolet (UV) band, extending partially to the soft X-rays. A fraction of the coronal emission illuminates the accretion disk, producing a rich reflection spectrum of fluorescent lines and other spectral features. This reflection component can be ionized, as changes in the ionization state of the disk determine the spectral features observed (e.g., Ross \& Fabian 2005; García \& Kallman 2010), and blurred and distorted by relativistic effects (e.g., Laor 1991; Crummy et al. 2006), if it originates close enough to the supermassive black hole; or it can be cold and neutral, if produced farther from the black hole in either the broad-line region or the torus (e.g., George \& Fabian 1991; Matt et al. 1991).

In a large fraction of Seyfert AGNs, a soft-excess component is also observed peaking near $1-2 \mathrm{keV}$. Its origin has been debated over the years. This soft excess was first believed to be the hard tail of UV blackbody emission from the accretion disk (Arnaud et al. 1985; Singh et al. 1985; Pounds et al. 1986; Magdziarz et al. 1998; Leighly 1999); however, this explanation 
Table 1

Observational Data Log for Mrk 509

\begin{tabular}{|c|c|c|c|c|c|}
\hline Telescope & Instrument & ObsID & Date & $\operatorname{Exp}(\mathrm{ks})$ & Counts $\left(10^{5}\right)$ \\
\hline NuSTAR & FPMA/B & 60101043002 & 2015 Apr 29 & 166 & 3.2 \\
\hline NuSTAR & FPMA/B & 60101043004 & 2015 Jun 2 & 37 & 0.6 \\
\hline Suzaku & XIS $0 / 3$ & 410017010 & 2015 May 1 & 47 & 2.1 \\
\hline
\end{tabular}

was ruled out, given that systems with very different accretion rates and/or masses would be characterized by the same blackbody temperature, which is not expected for an accretion disk (Gierliński \& Done 2004; Porquet et al. 2004; Piconcelli et al. 2005; Miniutti et al. 2009). The current models invoked to explain the soft excess tend to favor either Comptonization of UV photons or blurred ionized reflection. In the first case, the disk photons are Comptonized by a corona above the disk, which is optically thicker and cooler than the corona responsible for the primary X-ray emission (Czerny \& Elvis 1987; Jin et al. 2009; Middleton et al. 2009; Done et al. 2012). In the second case, the emission lines produced in the disk are relativistically blurred due to the proximity to the black hole (Fabian et al. 2002; Ross \& Fabian 2005; Crummy et al. 2006; García \& Kallman 2010; Walton et al. 2013).

The Seyfert type 1 galaxy Mrk 509 was one of the first AGNs to be studied in detail because it is luminous $\left(L_{\text {Bol }}=1.07 \times 10^{45} \mathrm{erg} \mathrm{s}^{-1}\right.$; Woo \& Urry 2002) and relatively nearby $(z=0.0344$; Fisher et al. 1995). The corresponding X-ray flux of $F_{\mathrm{x}}=(2-5) \times 10^{-11} \mathrm{erg} \mathrm{cm}^{-2} \mathrm{~s}^{-1}$ (Kaastra et al. 2011 ) is powered by a $1.4 \times 10^{8} M_{\odot}$ black hole (Peterson et al. 2004), which is accreting at $20 \%-30 \%$ of the Eddington rate (Petrucci et al. 2013). Excess soft ( $\lesssim 2 \mathrm{keV})$ emission above the extrapolation of the hard X-ray continuum was first identified by Singh et al. (1985). After Morini et al. (1987) detected the Fe line, improved X-ray instruments and detectors led to a full discussion of reflection features by Pounds et al. (1994).

An intense campaign of multiwavelength monitoring of Mrk 509 involving the X-ray observatories XMM-Newton and Suzaku has provided a detailed model for the observed set of soft X-ray absorption features, caused by differentially ionized warm absorbing gas (Kaastra et al. 2011). Portions of this gas phase have been observed to be outflowing at different velocities (Smith et al. 2007), including a component classed as an ultra-high-velocity outflow (Cappi et al. 2009). This campaign also resulted in the most complete study of the $\mathrm{Fe} \mathrm{K}$ complex of Mrk 509 to date, revealing a neutral narrow component and an ionized broad component. The latter has been interpreted as relativistic reflection from the inner regions of the accretion disk (Walton et al. 2013). Despite the presence of a warm absorber, Mrk 509 can still be considered a "bare" AGN. The intrinsic absorption is low enough that it does not complicate the determination of the reflection continuum (Walton et al. 2013).

Most of the previous analyses of Mrk 509 mentioned above have predominantly focused on understanding the physical details of the warm absorber, soft excess, and high-velocity outflows. Our emphasis is on the detection or nondetection of relativistic reflection features, namely, the Fe $\mathrm{K}$ complex and the Compton hump, which are likely to originate due to the reprocessing of hard X-rays in the innermost regions of the accretion disk. To date, observations of the hard X-ray component in which these signatures are most evident are quite limited, and the physical picture is accordingly subject to large and fundamental uncertainties (e.g., Petrucci et al. 2013; Ponti et al. 2013; Kaastra et al. 2014).

The low background, high sensitivity, and $\sim 3-79 \mathrm{keV}$ bandwidth (which captures the key reflection features) of the Nuclear Spectroscopic Telescope Array (NuSTAR; Harrison et al. 2013), together with the development of advanced relativistic reflection models such as relxill (Dauser et al. 2013; García et al. 2013, 2014), have revolutionized studies of X-ray reflection spectroscopy (e.g., Risaliti et al. 2013; Keck et al. 2015; Walton et al. 2014; Kara et al. 2017; Porquet et al. 2018). In this paper, we present an analysis of the first NuSTAR and last Suzaku observations of the bright AGN Mrk 509. Implementing a variety of X-ray spectral models, we investigate the origin of the soft excess and the possibility for relativistic ionized reflection in this source. Based on these fits, we present a theoretical discussion of the physical implications of two competing models to explain the soft excess: the warm corona and the relativistic reflection.

\section{Observational Data}

The first NuSTAR observations of Mrk 509 were taken during Cycle 1 of the Guest Observer Program on 2015 April 29 , with a total requested exposure time of $200 \mathrm{ks}$. A simultaneous Suzaku observation was performed with a $50 \mathrm{ks}$ exposure in order to provide low-energy coverage. The $\mathrm{NuSTAR}$ exposure was interrupted after $\sim 165 \mathrm{ks}$ due to a target-of-opportunity trigger. The remaining $\sim 35 \mathrm{ks}$ were taken roughly a month later on 2015 June 2 . A log with details of the observational data analyzed in this paper is shown in Table 1.

\subsection{NuSTAR Extraction}

The NuSTAR data are split over two ObsIDs, 60101043002 and 60101043004 , separated by roughly a month. We reduced these data following standard procedures using the NuSTAR Data Analysis Software (NuSTARDAS, v1.6.0) and instrumental calibration files from caldb v20160824. We first cleaned the unfiltered event files with NUPIPELINE. We used the standard depth correction, which significantly reduces the internal high-energy background, and removed passages through the South Atlantic Anomaly, again using standard filtering parameters. Source and background spectra/light curves and instrumental responses were then produced for both focal plane modules, FPMA and FPMB, using NUPRODUCTS. Source products were extracted from circular regions of radius $120^{\prime \prime}$, and the background was estimated from regions of blank sky on the same detector as Mrk 509. In order to maximize the signal-to-noise ratio $(\mathrm{S} / \mathrm{N})$, in addition to the standard "science" (mode 1) data, we also extracted the "spacecraft science" (mode 6) data following Walton et al. (2016). In this case, the mode 6 data provide $\sim 10 \%$ of the total $\sim 220 \mathrm{ks}$ good NuSTAR exposure. 

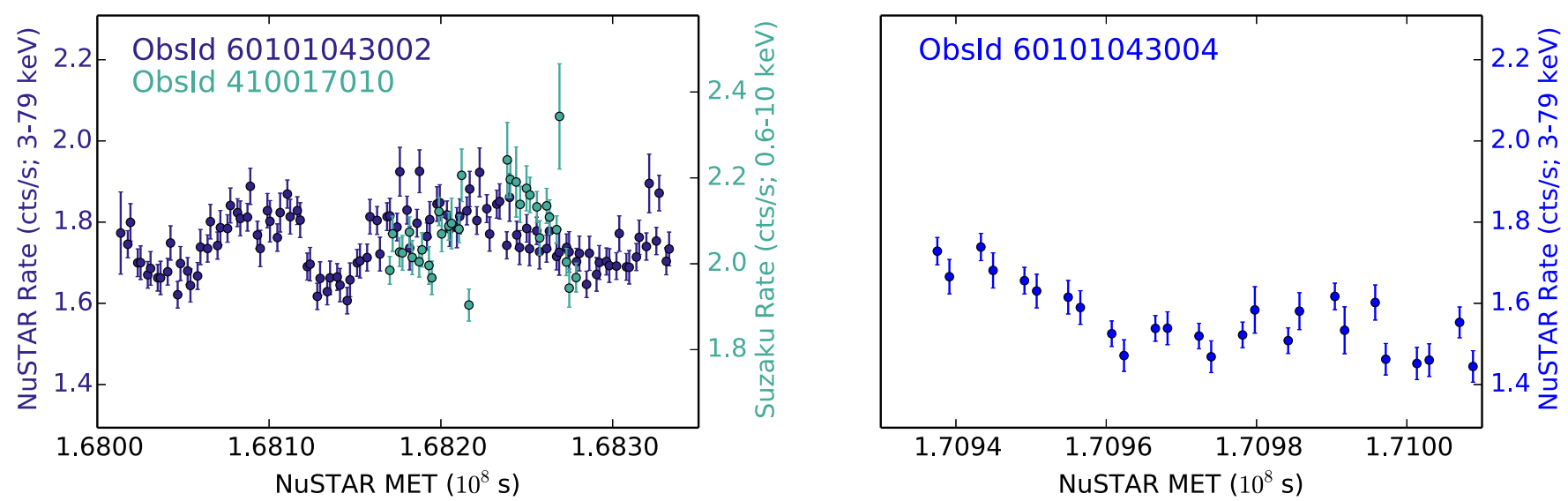

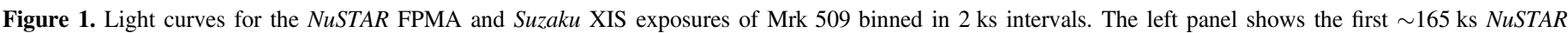

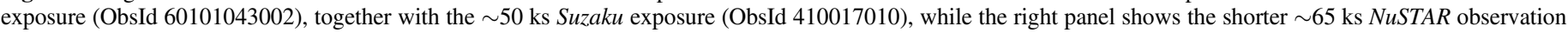
(ObsId 60101043004). The source shows a very stable count rate through the full range, with no obvious flares or dips.

\subsection{Suzaku Extraction}

The Suzaku data were reduced starting from the unfiltered event files and then screened by applying the standard selection criteria described in the Suzaku ABC guide. ${ }^{18}$ The source spectra were extracted from circular regions of 2.5 radius centered on the source, whereas background spectra were extracted from a region of the same size offset from the main target and avoiding the calibration sources. We generated the redistribution matrix file and the ancillary response file of the X-ray Imaging Spectrometer (XIS) with the xisrmfgen and xissimarfgen ftools, respectively. We selected the XIS data in both the $3 \times 3$ and $5 \times 5$ modes. The spectra were inspected for possible pileup contamination, and this possibility was excluded. The spectra of the front-illuminated XIS instruments (XIS 0 and XIS 3) were merged after checking that their fluxes were consistent. The data from the backilluminated XIS instrument, XIS 1, are not used due to its much lower sensitivity in the Fe $\mathrm{K}$ band and cross-calibration uncertainties with the front-illuminated XIS 0 and XIS 3.

\subsection{Light Curves and Time-averaged Spectra}

The light curves for the two NuSTAR and the Suzaku exposures are shown in Figure 1. The data were binned in $2 \mathrm{ks}$ intervals. The Suzaku exposure is simultaneous with the first and longer NuSTAR exposure. The light curves show a very similar level of variability, which in both cases is very weak ( $\sim 6 \%)$. This value corresponds to the normalized excess variance (Vaughan et al. 2003) that suppresses a possible rmsflux correlation usually found for unnormalized rms measures. The right panel of Figure 1 contains the light curve for the shorter $N u S T A R$ exposure taken roughly a month later. It shows a similar count rate with no significant variability; neither flares nor strong dips are detected. Spectra extracted from the two NUSTAR exposures imply consistency after visual inspection. We therefore combined these into a single spectrum taking advantage of the full $\sim 220 \mathrm{ks}$ exposure.

The final extracted total count spectra for NuSTAR's FPMA and FPMB and Suzaku's XIS instruments are shown in Figure 2. The shaded regions depict the corresponding backgrounds, which are well below the source counts up to $\sim 50 \mathrm{keV}$. We include Suzaku data in the $1-8 \mathrm{keV}$ range,

\footnotetext{
${ }^{18}$ http://heasarc.gsfc.nasa.gov/docs/suzaku/analysis/abc/
}

excluding the $1.7-2.5 \mathrm{keV}$ range due to calibration uncertainties. We ignore data below $1 \mathrm{keV}$ due to concerns over the quality of the calibration given molecular contamination of the XIS detectors; contamination reduces the effective area differently on each detector and as a function of off-axis angle (Koyama et al. 2007; Kettula et al. 2013), and it is expected to worsen over time (Madsen et al. 2017). NUSTAR data are included in the 3-79 keV range. The spectra were rebinned in order to oversample the instrument's resolution by a factor of 3 and ensure a minimum $\mathrm{S} / \mathrm{N}$ of 6 per bin.

\section{Spectral Analysis}

We simultaneously fit the two NuSTAR FPMA and FPMB spectra extracted from the full $\sim 220 \mathrm{ks}$ exposure together with the $\sim 50 \mathrm{ks}$ Suzaku spectrum. The fitting and statistical analysis presented here was carried out using the XSPEC package v12.9.0d (Arnaud 1996). A cross-normalization constant is included to account for differences in the flux calibration among all three instruments (i.e., FPMA, FPMB, and XIS). The fitted values are consistent with those previously reported by Madsen et al. (2015). All model parameter uncertainties are quoted to a $90 \%$ confidence level.

Figure 2 (right) shows the data-to-model ratio of these observations when fitted with a simple absorbed power-law model (i.e., TBabs*pow). The TBabs component is used to describe the Galactic absorption (see Section 3.1). The typical signatures of X-ray reflection off optically thick material are evident in the spectrum: the fluorescent iron emission near $6.4 \mathrm{keV}$, the iron $\mathrm{K}$ edge near $7 \mathrm{keV}$, and the Compton hump peaking at $\sim 25 \mathrm{keV}$. Both instruments satisfactorily agree in the shape and intensity of the iron emission. In the absence of relativistic effects, these features are well described by the reprocessing of the X-rays in a relatively cold and neutral material located far away from the central region, possibly at the broad-line region (e.g., Costantini et al. 2016; Nardini et al. 2016) or even at the torus (e.g., Yaqoob et al. 2007; Murphy \& Yaqoob 2009; Marinucci et al. 2018).

The nature of the soft excess in Suzaku's bandpass, however, is not yet very well established. As we shall show next, the particular choice of components used to model the soft excess has an important effect in the modeling of the reflected spectrum and, in fact, leads to different interpretations for this system. We will then present fits with two different scenarios 

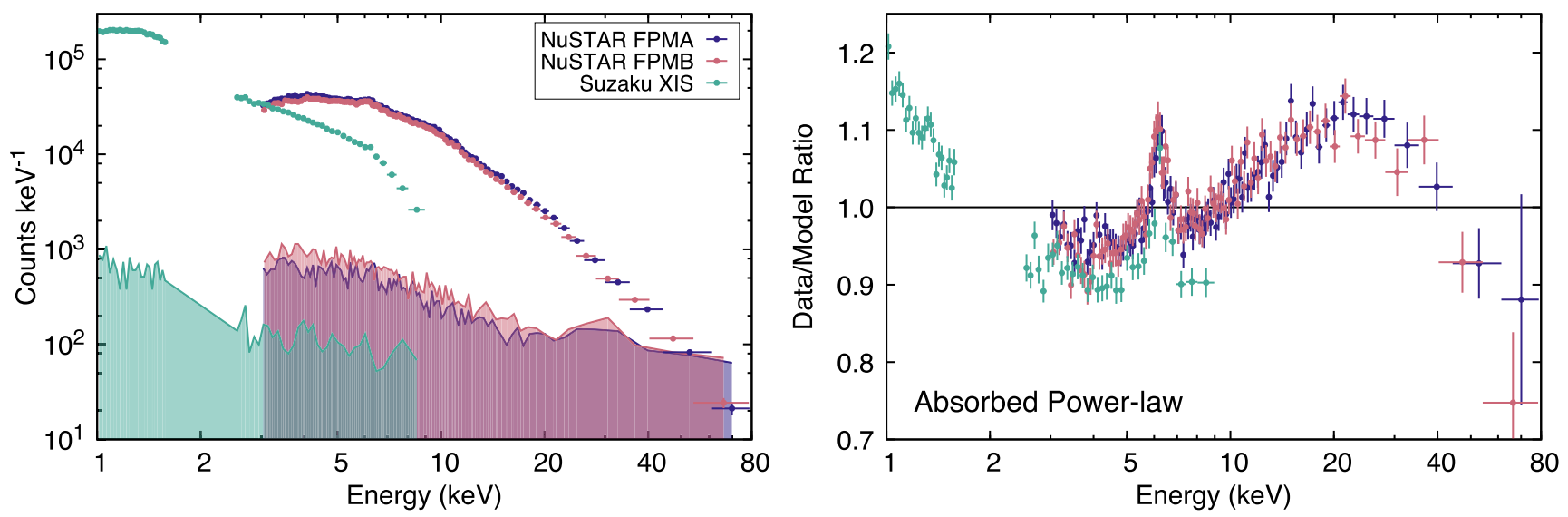

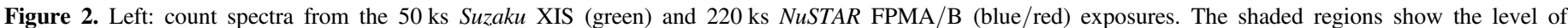

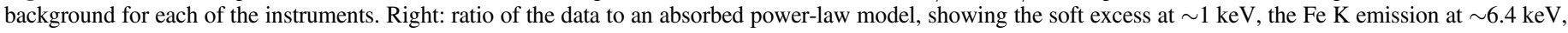
and the Compton hump at $\sim 20-30 \mathrm{keV}$.

and later discuss the physical interpretation and implications for each one.

\subsection{Approach 1: Fitting the Soft Excess with a Warm Corona}

In their analysis of the XMM-Newton and INTEGRAL campaign, Petrucci et al. (2013) proposed that the clearly observed soft excess in Mrk 509 is due to the presence of a warm corona, which they reproduced using a Comptonization model. This corona can then be visualized as a warm $\left(k T_{\mathrm{e}} \sim 0.5-1 \mathrm{keV}\right)$ but optically thick $(\tau \sim 10-20)$ atmosphere sitting on top of the accretion disk. This extended, slablike corona is much colder than the centrally located and possibly spherical corona responsible for the power-law continuum emission that extends to high energies. The emission of the hot corona was fitted with a second Comptonization model, with a higher coronal temperature $\left(k T_{\mathrm{e}} \sim 100 \mathrm{keV}\right)$ and lower optical depth $(\tau \sim 0.5)$.

We adopted the prescription of Petrucci et al. (2013) to fit the soft excess. For this, we implemented two Comptonization components using the nthComp model (Zdziarski et al. 1996; Życki et al. 1999) with the required parameters to reproduce the power-law continuum (hot corona) and soft excess (warm corona). The hot corona component is characterized by a slope of $\Gamma \sim 1.84$ and a relatively low electron temperature of $k T_{\mathrm{e}} \sim 30 \mathrm{keV}$. The temperature of the seed photons for this component cannot be constrained, and it is thus fixed to a relatively low value $\left(k T_{\mathrm{BB}}=100 \mathrm{eV}\right)$. The warm corona component is characterized by a very soft continuum fixed at $\Gamma=2.5$ and a much colder electron temperature, $k T_{\mathrm{e}} \sim 0.4-0.5 \mathrm{keV}$, as well as a much lower temperature for the seed photons, fixed at $k T_{\mathrm{BB}}=3 \mathrm{eV}$. The parameters held fixed in these two components cannot be constrained with the current data set, likely due to the lack of data below $1 \mathrm{keV}$. Their values were chosen following the best-fit results of Petrucci et al. (2013). The intrinsic galactic absorption in this system is modeled using the TBabs model with the corresponding abundances as set by Wilms et al. (2000). This model automatically implements the Verner et al. (1996) photoelectric cross sections. We freeze the column density to $N_{\mathrm{H}}=4.25 \times 10^{20} \mathrm{~cm}^{-2}$ (Kalberla et al. 2005) and the source redshift to $z=0.035$.

A data-to-model ratio plot of the fit using these models for the continuum is shown in the top panel of Figure 3. These two

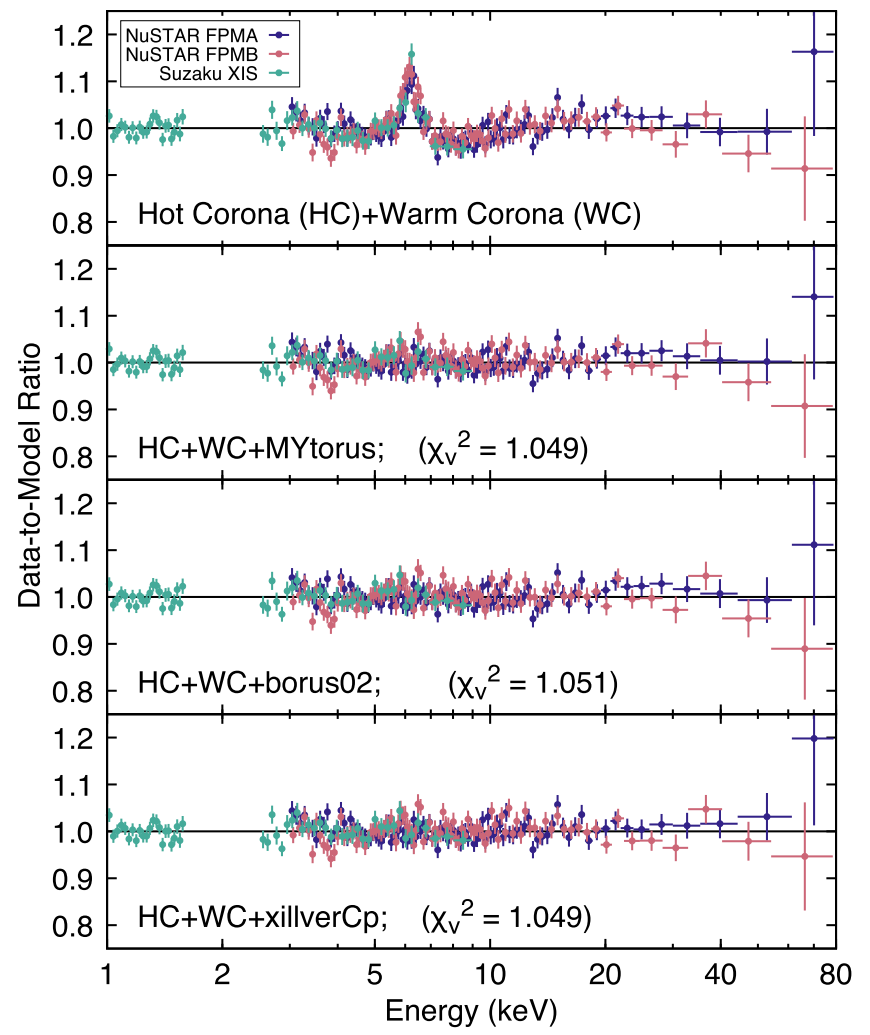

Figure 3. Data-to-model ratios for the fits with the warm corona and different models for the distant reflection.

Comptonization components, which are independent from one another, provide a good fit to both the continuum and the soft excess, and the only obvious residuals are those from the Fe K fluorescence emission due to X-ray reflection.

The residuals that remain after fitting the continuum can be well fitted with a distant reflection model component, in which the gas is assumed to be either completely neutral or at a very low ionization stage, and no relativistic effects are included. We have tested this idea by implementing three different (nonrelativistic) reflection models, namely, MYtorus, borus02, and xillvercp, which we describe below. The residuals of these fits are shown in the last three panels of Figure 3, and the best-fit values are summarized in Table 2. 
Table 2

Best-fit Parameters and Fit Statistics for the Three Models Featuring a Warm Corona Prescription

\begin{tabular}{|c|c|c|c|c|c|}
\hline Description & Component & Parameter & Model 1.1 & Model 1.2 & Model 1.3 \\
\hline Galactic absorption & TBabs & $N_{\mathrm{H}}\left(10^{20} \mathrm{~cm}^{-2}\right)$ & 4.25 & 4.25 & 4.25 \\
\hline $\begin{array}{l}\text { Hot corona } \\
\text { Redshift } \\
\text { Warm corona }\end{array}$ & $\begin{array}{l}\text { nthComp } \\
\text { nthComp } \\
\text { nthComp } \\
\text { nthComp }\end{array}$ & $\begin{array}{c}k T_{\mathrm{BB}}(\mathrm{eV}) \\
z \\
k T_{\mathrm{BB}}(\mathrm{eV}) \\
\Gamma\end{array}$ & $\begin{array}{c}100 \\
0.035 \\
3 \\
2.5\end{array}$ & $\begin{array}{c}100 \\
0.035 \\
3 \\
2.5\end{array}$ & $\begin{array}{c}100 \\
0.035 \\
3 \\
2.5\end{array}$ \\
\hline Hot corona & $\begin{array}{l}\text { nthComp } \\
\text { nthComp } \\
\text { nthComp } \\
\text { nthComp } \\
\text { nthComp }\end{array}$ & $\begin{array}{c}\Gamma \\
k T_{\mathrm{e}}^{\mathrm{HC}}(\mathrm{keV}) \\
N_{\mathrm{HC}}\left(10^{-2}\right) \\
k T_{\mathrm{e}}^{\mathrm{WC}}(\mathrm{keV}) \\
N_{\mathrm{WC}}\left(10^{-2}\right)\end{array}$ & $\begin{array}{c}1.84 \pm 0.01 \\
26_{-4}^{+6} \\
1.33 \pm 0.02 \\
0.39 \pm 0.07 \\
0.34 \pm 0.07\end{array}$ & $\begin{array}{c}1.83 \pm 0.01 \\
29_{-4}^{+6} \\
1.32 \pm 0.02 \\
0.40 \pm 0.07 \\
0.35 \pm 0.06\end{array}$ & $\begin{array}{c}1.84 \pm 0.01 \\
29_{-4}^{+6} \\
1.29 \pm 0.02 \\
0.50_{-0.08}^{+0.05} \\
0.37 \pm 0.07\end{array}$ \\
\hline Neutral reflection & $\begin{array}{l}\text { MYtorus } \\
\text { MYtorus }\end{array}$ & $\begin{array}{c}N_{\mathrm{H}}\left(10^{24} \mathrm{~cm}^{-2}\right) \\
N_{\mathrm{MY}}\end{array}$ & $\begin{array}{l}1.06 \pm 0.14 \\
1.25 \pm 0.12\end{array}$ & $\begin{array}{l}\cdots \\
\cdots\end{array}$ & $\begin{array}{l}\cdots \\
\cdots\end{array}$ \\
\hline Neutral reflection & $\begin{array}{l}\text { borus } 02 \\
\text { borus02 }\end{array}$ & $\begin{array}{c}N_{\mathrm{H}}\left(10^{24} \mathrm{~cm}^{-2}\right) \\
N_{\mathrm{B}}^{\mathrm{b}}\end{array}$ & $\begin{array}{l}\cdots \\
\cdots\end{array}$ & $\begin{array}{l}1.17 \pm 0.20 \\
0.93 \pm 0.08\end{array}$ & $\begin{array}{l}\cdots \\
\cdots\end{array}$ \\
\hline Neutral reflection & $\begin{array}{l}\text { xillverCp } \\
\text { xillverCp }\end{array}$ & $\begin{array}{c}i(\mathrm{deg}) \\
N_{\mathrm{Xi}}\left(10^{-2}\right)^{\mathrm{b}}\end{array}$ & $\begin{array}{l}\cdots \\
\cdots\end{array}$ & $\begin{array}{l}\cdots \\
\cdots\end{array}$ & $\begin{array}{c}89^{\mathrm{a}} \\
0.25_{-0.01}^{+0.02}\end{array}$ \\
\hline Cross-normalization & $\begin{array}{c}\text { NuSTAR FPMB } \\
\text { Suzaku XIS }\end{array}$ & $\begin{array}{c}C_{\mathrm{FPMB}} \\
C_{\mathrm{XIS}}\end{array}$ & $\begin{aligned} 1.026 & \pm 0.005 \\
0.95 & \pm 0.04\end{aligned}$ & $\begin{aligned} 1.026 & \pm 0.005 \\
0.95 & \pm 0.03\end{aligned}$ & $\begin{aligned} 1.026 & \pm 0.005 \\
0.95 & \pm 0.03\end{aligned}$ \\
\hline & $\begin{array}{c}\chi^{2} \\
\nu \\
\chi_{\nu}^{2}\end{array}$ & & $\begin{array}{c}1799.5 \\
1716 \\
1.049\end{array}$ & $\begin{array}{c}1804.0 \\
1716 \\
1.051\end{array}$ & $\begin{array}{c}1800.4 \\
1716 \\
1.049\end{array}$ \\
\hline
\end{tabular}

Notes. Model 1.1: TBabs* (nthComp+nthComp+MYtorus); Model 1.2: TBabs* (nthComp+nthComp+borus02); and Model 1.3: TBabs* (nthComp $+n t h$ Comp+xillverCp). The parameters listed in the first block were assumed fixed at the same value in all models.

${ }^{a}$ Parameter pegged at its maximum value.

${ }^{\mathrm{b}}$ Model normalizations in $10^{20}$ photons $\mathrm{cm}^{-2} \mathrm{~s}^{-1}$.

Model 1.1. The MYtorus reflection model (Murphy \& Yaqoob 2009) calculates the attenuation in the line of sight of the X-rays produced by a central source, together with the scattered continuum, and the fluorescence emission from neutral iron and nickel, assuming a toroidal geometry. In this model, the X-ray source emits a power-law continuum with no cutoff at high energies. All elemental abundances are at their solar values. In our fit, all of the parameters of the transmitted and scattered components are tied to each other. The photon index is linked to the one from the hot corona component. The inclination is fixed to $60^{\circ}$, as it has no appreciable effect on the fit. Thus, the column density and normalization are the only free parameters.

Model 1.2. The borus 02 model (Baloković et al. 2018) is similar to MYtorus in nature but more flexible, as it provides additional tunable spectral parameters, such as the high-energy cutoff in the intrinsic continuum, the torus covering factor, and the relative abundance of iron. The approximately toroidal geometry assumed for the model employed here ${ }^{19}$ is the same as in the popular model by Brightman \& Nandra (2011), but the model is updated, expanded, and corrected for known issues as described in Liu \& Li (2015) and Baloković et al. (2018). Like MYtorus, borus 02 allows us to model the average column density of the torus separately from the line-of-sight column density through the spectral shape of the reflection from material outside of our line of sight. As before, the inclination is degenerate in our fits, which allows us to fix it at $60^{\circ}$. Again,

\footnotetext{
$\overline{19}$ We used table model borus02_afe1p00_v161220.fits, available at www.astro.caltech.edu/ mislavb/download.
}

the photon index is linked to the one describing the Comptonized emission of the hot corona. Furthermore, the covering fraction is fixed to $50 \%$, and the iron abundance is set to its solar value. Finally, the simple relation $E_{\text {cut }} \sim(2-3) k T_{\mathrm{e}}$ is used to link the cutoff at high energies with the electron temperature of the hot corona (e.g.; Petrucci et al. 2001; García et al. 2015). While this is a crude approximation that depends on the combination of temperature, optical depth, and geometry, we found that it is adequate for this model fit. First, the value of $E_{\text {cut }}$ is unconstrained when set free to vary in the borus 02 model, while all other model parameters remain unchanged. Furthermore, replacing the Comptonization continuum with a simple cutoff power-law model provides an identical fit with $E_{\text {cut }}=95-175 \mathrm{keV}$ (90\% confidence), consistent with $E_{\text {cut }} \sim 3 k T_{\mathrm{e}}$. Thus, we use this relation to link the cutoff in the reflection model with the temperature of the hot corona. As in the case of Model 1.1, the only free parameters are the column density and normalization.

Model 1.3. We reproduced the observed residuals with our ionized reflection model xillverCp. This particular flavor of the model computes the reflected spectrum using an illumination continuum produced by the Comptonization model nthComp, which is a more physically consistent treatment than the standard and commonly used power-law continuum with an exponential cutoff. While xillvercp has a more accurate treatment of the reflection by self-consistently solving the ionization balance and radiative transfer, the geometrical considerations are much more simplistic than in MYtorus or borus02. In xillverCp, a single-zone, plane-parallel slab 
is assumed. Despite this approximation, this model also provides a satisfactory fit to the data (Figure 3, bottom panel). As before, the slope of the illumination is fixed to that in the hot corona model. Moreover, we fixed the ionization parameter, defined as the ratio of the ionizing flux to the gas density $\left(\xi=4 \pi F_{\mathrm{x}} / n_{\mathrm{e}}\right)$, to its minimum value in the model in order to mimic reflection off neutral gas $\left(\log \xi / \mathrm{erg} \mathrm{cm} \mathrm{s}^{-1}=\right.$ $0)$. We assumed a solar abundance of iron. In this case, the inclination has a small but noticeable effect on the fit, with the best-fit value pegged at its maximum $\left(i=89^{\circ}\right)$. Fixing the inclination to a more reasonable value (e.g., $i=60^{\circ}$ ) worsens the fit significantly $\left(\Delta \chi^{2} \sim 60\right)$, due to strong residuals at high energies and near the Fe $\mathrm{K}$ band. While this could be taken as the possible presence of a broad $\mathrm{Fe}$ line component, its statistical significance is low. Moreover, given the simplicity of the xillverCp model in its geometrical considerations, we do not interpret the derived inclination as a meaningful estimate. In addition to the inclination, the normalization is the only other free parameter in this fit.

From a statistical point of view, these three models are indistinguishable. Only very small differences in the goodness of the fit are apparent in the bottom of Table 2. From these, the fit with the borus 02 model (Model 1.2) is slightly worse, but with a marginal increase in $\chi^{2}$ of $\sim 4$ when compared to the other two. From the ratio plots shown in Figure 3, it appears that these three models perform equally well in describing the data. Despite some small differences, these three fits share the same relevant aspects. First, no inner-disk (relativistic) reflection is required in any of the fits, as no significant residuals remain in the $\mathrm{Fe} \mathrm{K}$ region. Second, the electron temperature of the hot corona is relatively low $\left(k T_{\mathrm{e}}^{\mathrm{HC}} \sim\right.$ $30 \mathrm{keV}$ ), which suggests a low-energy cutoff in the continuum. Finally, the electron temperature of the warm corona is similar in all the fits at $k T_{\mathrm{e}}^{\mathrm{WC}} \sim 0.4-0.5 \mathrm{keV}$, which is consistent with values previously derived by Petrucci et al. (2013).

\subsection{Approach 2: Fitting the Soft Excess with Relativistic Reflection}

Another approach that has been proposed in the past to explain the soft excess in AGNs is relativistic reflection (e.g., Crummy et al. 2006; Fabian et al. 2009; Nardini et al. 2012; Walton et al. 2013). As the X-rays from the central source illuminate the inner regions of the accretion disk, the reflected or reprocessed radiation displays a spectrum rich in fluorescence lines and other atomic features. This spectrum is particularly populated with emission lines in the low-energy range $(\lesssim 1 \mathrm{keV})$, where most of the K-shell transitions from low- $Z$ elements occur. As the reprocessing is produced near the supermassive black hole, relativistic effects will blur and skew all the atomic features, effectively smoothing the entire reflected spectrum. As a result, this component can in fact produce enough flux at low energies to explain the observed soft excess. Furthermore, we have recently shown that this effect is further enhanced if the density of the reflecting material lies above the typically assumed value of $n_{\mathrm{e}}=10^{15} \mathrm{~cm}^{-3}$, due to the extra heating produced by the increased free-free emission (García et al. 2016).

In order to test this approach, we replaced the warm corona component with a relativistic reflection component. For this, we implemented our model relxilld, which describes both the incident Comptonized continuum and the reflection spectra calculated with our code xillver (García \& Kallman 2010;
García et al. 2013) in the case of a high-density gas (xillverD; García et al. 2016), taking into account all of the relativistic effects (Dauser et al. 2013; García et al. 2014). While the relxillD model has the advantage of providing the gas density as a free parameter, one limitation is that the illumination continuum assumed is a power-law spectrum with an $e$-folded cutoff fixed at $300 \mathrm{keV}$ (instead of the Comp-

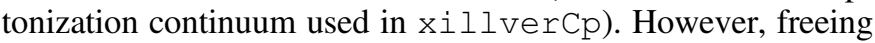
up the cutoff energy will only introduce a significant effect in the fit if the curvature imprinted in the power-law continuum falls within the covered bandpass and can be detected given the instrument's $\mathrm{S} / \mathrm{N}$.

In this fit, from here on Model 2, the distant (nonrelativistic) reflection is still modeled with xillverCp, as in Model 1.3. For the relativistic reflection, we use the specific flavor of relxilld, namely, relxilllpD, in which a lamppost geometry is assumed for the hot corona (Dauser et al. $2013,2016)$ that is self-consistently linked with the reflected continuum. The slopes of both the distant and inner-disk reflection components are tied to that in the hot corona, as well as the electron temperature in xillverCp. The inclination of the system is tied among the two reflection components. Unlike the previous fits with Models 1.1-1.3, in this case, the electron temperature of the hot corona is loosely constrained. Fixing $k T_{\mathrm{e}}=30 \mathrm{keV}$ (similar to the value found with the fits in Section 3.1) results in a significantly worse fit (with $\chi^{2}$ increasing by $\sim 40$ ) and obvious residuals in excess at high energies. This indicates that this particular fit prefers a cutoff at much larger energies. Adopting once again the simple approximation $E_{\mathrm{e}}=3 k T_{\mathrm{e}}$, we fixed the electron temperature of the hot corona at $100 \mathrm{keV}$ (i.e., one-third of the cutoff energy of $300 \mathrm{keV}$ in the relxillipD component). The best-fit parameters are summarized in Table 3.

In terms of fit statistics, the relativistic reflection prescription reproduces the data similarly well as the warm corona prescription from Model 1. The fit with Model 2 is marginally worse, with an increase of $\Delta \chi^{2} \sim 6-10$, despite using three more free parameters. It is, however, unclear if any of these fits is preferred on statistical grounds. The model components and residuals of the fits with the two scenarios (Models 1.3 and 2) are compared in Figure 4. The two models are almost identical in the band covered by the data, with the largest differences occurring around 30-60 keV for Model 2. These residuals are possibly due to the fact that the reflection model used here was calculated using an $e$-folded power-law illumination spectrum with a high-energy cutoff fixed at $300 \mathrm{keV}$, rather than a proper Comptonization continuum. On the other hand, we also note that Model 2 allows for a softer continuum $(\Gamma=1.96)$ than Model $1.3(\Gamma=1.84)$, which can also affect the way the model fits the rollover at high energies.

Despite its statistical match, the relativistic reflection component (relxilllpD) requires extreme parameters, i.e., low coronal height $\left(h=1.53_{-0.25}^{+0.01} R_{\mathrm{Hor}}\right)$ and close to maximum spin $\left(a_{*}>0.993\right)$, together with a large gas density $\left(\log n_{\mathrm{e}}\right.$ $\left./ \mathrm{cm}^{-3}>18.2\right)$. This configuration results in a soft and featureless spectrum with a strong broad emission at low energies, which is required to fit the soft excess.

\section{Discussion}

In the previous section, we presented several model fits to the observational data of Mrk 509. These models are based on 
Table 3

Best-fit Parameters and Fit Statistics for the Model Featuring Relativistic Reflection

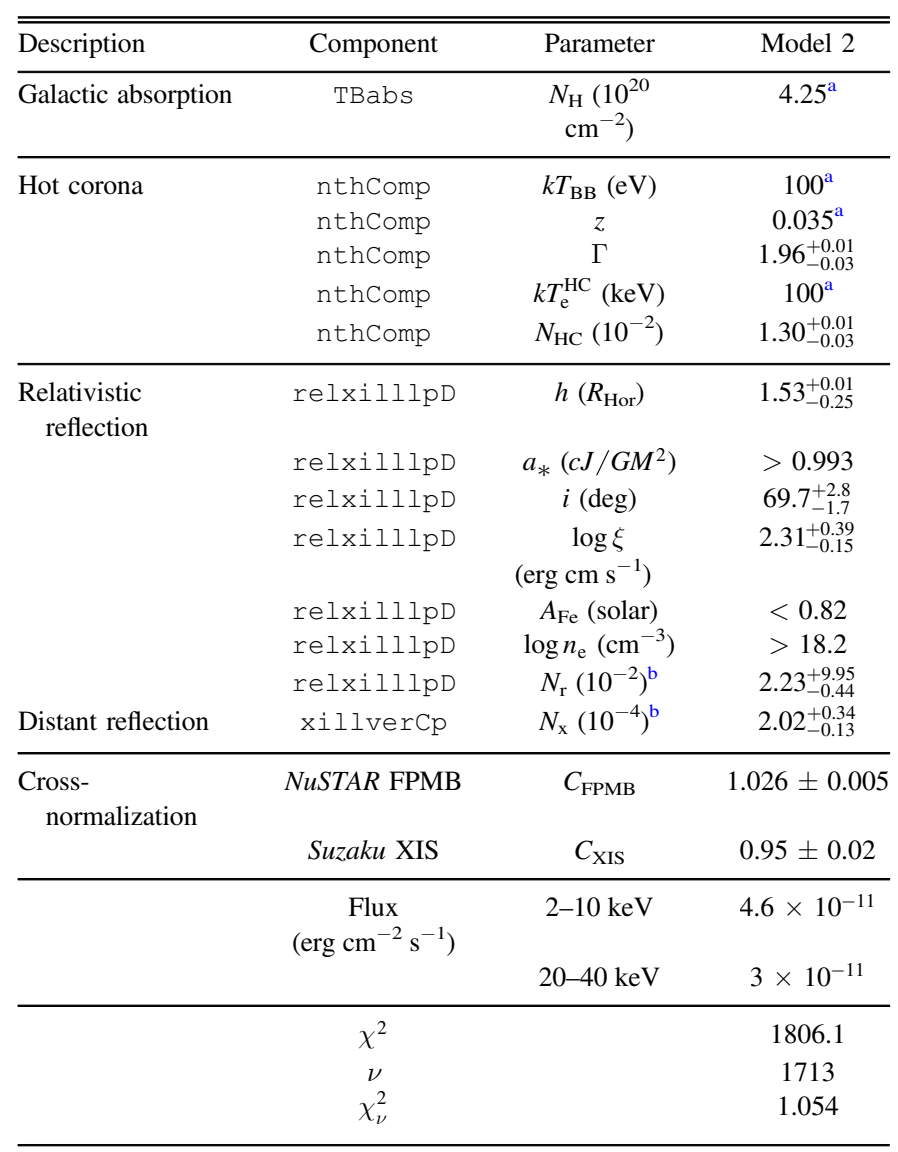

Notes. Model 2: TBabs* (nthComp+relxilld+xillverCp).

${ }^{\text {a }}$ Parameter fixed to the quoted value.

${ }^{\mathrm{b}}$ Model normalizations in $10^{20}$ photons $\mathrm{cm}^{-2} \mathrm{~s}^{-1}$.

two different scenarios to explain the origin of the soft excess in the spectrum: the warm corona and the relativistic reflection picture. In either case, strong signatures of reflection are observed (i.e., $\mathrm{Fe} \mathrm{K}$ emission and $\mathrm{K}$ edge, plus a Compton hump). This signal is consistent with low-ionization reflection from a structure located at a farther distance such that no relativistic effects are observed. Models for Compton-thick AGNs (MYtorus and borus02; Models 1.1 and 1.2) and nearly neutral reflection from a single plane-parallel slab (xillverCp; Model 1.3) all provide equally good fits to the data. This implies that the geometrical considerations for the distribution of gas in the line of sight are relatively unimportant. Moreover, we notice that no other components are required to fit the $\mathrm{Fe} \mathrm{K}$ emission, while Ponti et al. (2013) reported both a narrow $(\sigma=0.027 \mathrm{keV})$ and a resolved $(\sigma=0.22 \mathrm{keV})$ Gaussian feature for the $\mathrm{Fe} \mathrm{K}$ line in their analysis of previous Chandra grating data. However, these two components were unresolved in their XMM-Newton and Suzaku data and likewise are expected to be unresolved in our $X M M$ Newton and NuSTAR data. This is possibly the reason why Models 1.1-1.3 are able to reproduce the spectral features without any additional components. Weak ionized emission features were also reported by Ponti et al. (2013), which could be attributed to Fe XXV-XXVI. We do not find evidence for these additional components, possibly due to the lower $\mathrm{S} / \mathrm{N}$ of our data.

For the sake of comparison, we will now focus on the fits performed with Models 1.3 (warm corona) and 2 (relativistic reflection at high densities) and discuss the physical implications of each scenario.

\subsection{Implications of the Warm Corona Model}

In the warm corona model, the soft emission observed in excess of the hard power-law continuum originates in Comptonization of thermal disk photons into a warm $\left(T \sim 0.5-1 \mathrm{keV}\right.$ or $\left.\sim 0.6-1.2 \times 10^{7} \mathrm{~K}\right)$ and optically thick $\left(\tau_{\mathrm{T}} \sim 10-20\right)$ corona (Walter \& Fink 1993; Magdziarz et al. 1998; Done et al. 2012). This warm corona has been described as a slab sitting on top of a passive accretion disk covering roughly $10-20 R_{\mathrm{g}}$ of the inner region (e.g., Petrucci et al. 2013). One argument that favors this scenario is the observed correlation between the optical-UV and the soft X-ray emission (Mehdipour et al. 2011). As shown in our fits to Models 1.1-1.3, the warm corona model provides a satisfactory description of the data in combination with a distant reflection component, without the requirement of relativistic reflection.

In this case, the temperature of the hot corona (the one responsible for the hard power-law continuum) is found to be relatively low $\left(k T_{\mathrm{e}} \sim 25-35 \mathrm{keV}\right.$ or $\left.\sim 3-4 \times 10^{8} \mathrm{~K}\right)$. While low coronal temperatures were not common in earlier studies of AGNs (e.g., Marinucci et al. 2016), several recent NuSTAR measurements have reported relatively cold coronae, namely, $\sim 50 \mathrm{keV}$ (IC 4329A; Brenneman et al. 2014), $\sim 25 \mathrm{keV}$ (MCG -05-23-016; Baloković et al. 2015), 40 keV (NGC 5548; Ursini et al. 2015), $12 \mathrm{keV}$ (GRS 1734-292; Tortosa et al. 2017), $\sim 35 \mathrm{keV}$ (IRAS 05189-2524; Xu et al. 2017), and $\sim 15 \mathrm{keV}$ (Ark 564; Kara et al. 2017). Moreover, Ricci et al. (2017) also reported a handful of sources with low cutoff energies fitting $e$-folded power-law models to sources from the Swift/BAT sample and found that those sources appear to be the ones with the highest Eddington ratios. Meanwhile, Tortosa et al. (2018) reported more reliable coronal temperatures for a sample of AGNs by implementing thermal Comptonization models in which most of the sources are found to have coronal temperatures below $\sim 60 \mathrm{keV}$.

While the warm corona model has been successfully used in several other sources (see Petrucci et al. 2018 and references therein), its physical origin and implications have yet to be fully explained. Czerny et al. (2003) argued that a warm Comptonizing skin on top of the accretion disk under radiation pressure instabilities could explain the observed X-ray spectra from quasars and narrow-line Seyfert AGNs. Różańska et al. (2015) investigated the properties of such a corona by solving the radiative transfer for a gray atmosphere. More recently, Petrucci et al. (2018) presented a theoretical discussion to explain the warm corona based on simple photon conservation arguments, concluding that most of the energy dissipation takes place in the warm corona rather than in the accretion disk. Meanwhile, Kaufman et al. (2018) proposed that bulk Comptonization from turbulence due to magnetorotational instabilities can explain the warm corona. These authors argued, however, that this picture is only applicable to systems with high accretion rates, possibly of an order or larger than the Eddington limit. Crucially, all of these theoretical studies share the same fundamental limitation: they neglect the effects of 

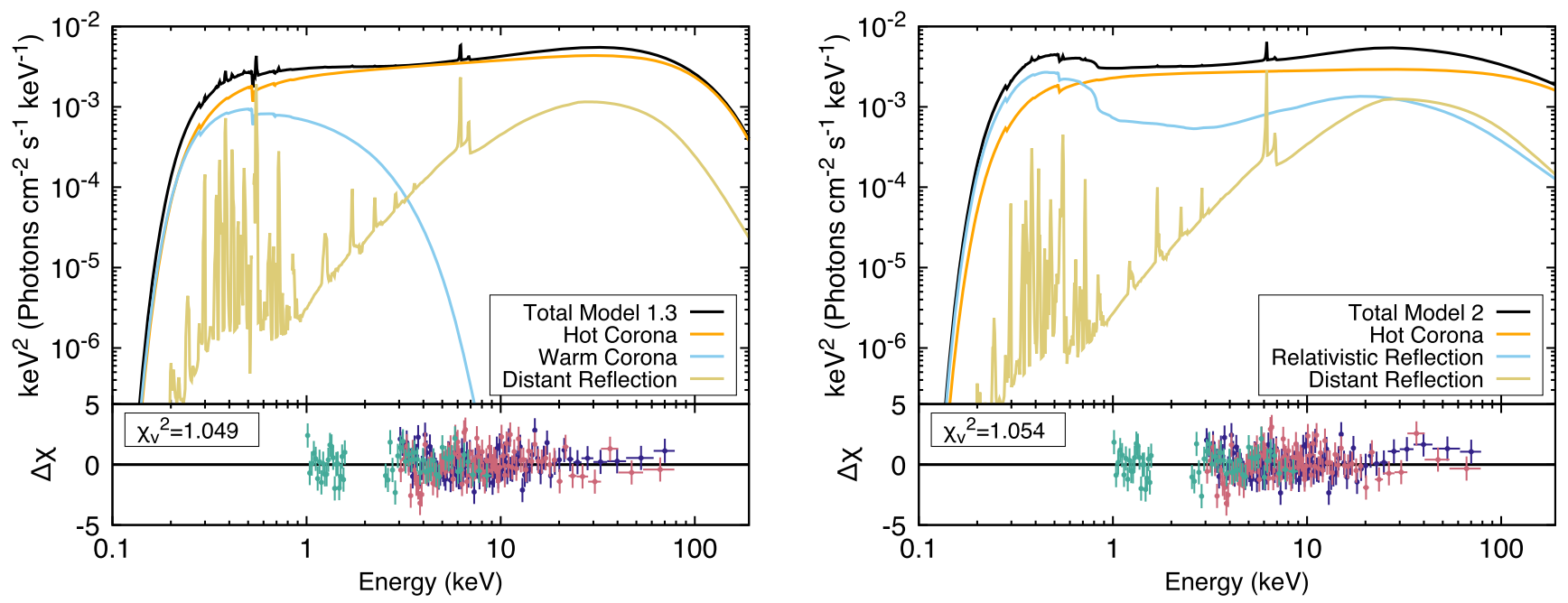

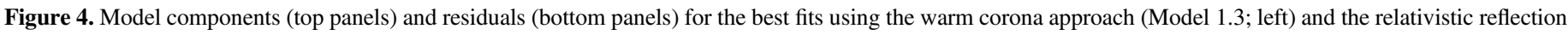
approach (Model 2; right) to describe the soft excess observed in Mrk 509.

atomic photoelectric absorption, which is likely to be a dominant process in optically thick atmospheres.

It is interesting to describe the basic properties of the warm corona based on the average quantities obtained from fits to observational data (e.g., $\tau_{\mathrm{T}} \sim 10$ and $k T_{\mathrm{e}} \sim 0.5 \mathrm{keV}$; Petrucci et al. 2018). The vertical extension of this corona can be estimated as

$$
z=\tau_{\mathrm{T}} /\left(\sigma_{\mathrm{T}} n_{\mathrm{e}}\right),
$$

where $\sigma_{\mathrm{T}}=6.65 \times 10^{-25} \mathrm{~cm}^{2}$ is the Thomson cross section and $n_{\mathrm{e}}$ is the electron density. Therefore, $z \sim 1.5 \times$ $10^{24} \tau_{\mathrm{T}} n_{\mathrm{e}}^{-1} \mathrm{~cm}$, or, in units of the gravitational radius, $R_{g}=$ $G M / c^{2} \approx 1.5 \times 10^{13}\left(M_{8} / M_{\odot}\right) \mathrm{cm}$,

$$
z / R_{g} \sim 10^{11}\left(M_{8} / M_{\odot}\right)\left(\tau_{\mathrm{T}} / n_{\mathrm{e}}\right)
$$

where $G$ is the gravitational constant, $c$ is the speed of light, and $M_{8}=10^{8} M_{\odot}$. In the case of Mrk 509, $M_{8} / M_{\odot} \sim 1$ (Peterson et al. 2004), and thus the density must be of the order of $n_{\mathrm{e}} \sim 10^{12} \mathrm{~cm}^{-3}$ or higher for the warm corona to have a reasonable $\left(z \sim R_{g}\right)$ geometrical thickness. Moreover, for sources with $M \sim 10^{5}-10^{6} M_{\odot}$, this estimate implies densities for the warm corona of the order of the typical values used for the accretion disk atmosphere in X-ray reflection calculations (e.g., $n_{\mathrm{e}} \sim 10^{15} \mathrm{~cm}^{-3}$; Ross \& Fabian 2005; García \& Kallman 2010).

One requirement for the warm corona scenario is to ensure that electron scattering is the dominant source of opacity. However, Krolik \& Kallman (1984) showed that for an optically thin gas under coronal ionization equilibrium (CIE), the photoelectric opacity dominates the soft band for $T \sim 10^{6} \mathrm{~K}$, and even at $T \sim 10^{7} \mathrm{~K}$, it is comparable to the Thomson opacity at $\sim 1 \mathrm{keV}$ (see their Figure 1 ). We have tested this argument by computing simulations for an optically thick plasma under CIE using the latest version of the XSTAR code (Kallman \& Bautista 2001), with the appropriate parameters that describe a warm corona: $n_{\mathrm{e}}=10^{12} \mathrm{~cm}^{-3}$, $\tau_{\mathrm{T}}=6.65$ (corresponding to the maximum column allowed by the model, $N_{\mathrm{H}}=10^{25} \mathrm{~cm}^{-2}$ ), $L_{\mathrm{x}}=10^{46} \mathrm{erg} \mathrm{s}^{-1}$ (which is in fact larger than the value typically measured for this source), and cosmic abundances. The incident spectrum is assumed to be a blackbody at the given gas temperature. Figure 5 (left) shows the resulting photoelectric opacity as a function of energy for different gas temperatures, in comparison with the Thomson opacity for electron scattering, $\sigma_{\mathrm{T}}$. This demonstrates that even in the optically thick case, photoelectric opacity dominates over a wide range of energies, particularly around or above $1 \mathrm{keV}$, for the range of temperatures required by the warm corona, i.e., $k T \sim 0.1-1 \mathrm{keV}\left(T \sim 10^{6}-10^{7} \mathrm{~K}\right)$. The right panel in Figure 5 shows the transmitted spectra for these two CIE calculations. At $T=10^{6} \mathrm{~K}$, the original disk blackbody emission is heavily absorbed and modified, with strong photoabsorption at almost all energies and no emission above $\sim 300 \mathrm{eV}$. The situation is better at $T=10^{7} \mathrm{~K}$, although strong absorption is still present, particularly around 0.1 and $1 \mathrm{keV}$. We found in general that for electron scattering to be a dominant source of opacity, temperatures well above $10^{7} \mathrm{~K}$ are required.

Another possibility is to instead invoke a gas under photoionization equilibrium (PIE), since a radiation field strong enough can be responsible for stripping most of the ions and thus considerably reducing the total photoelectric opacity. This is in fact relevant, since one expects the ionization of the warm corona to be fairly large, from simple arguments. We start by using the standard definition of the ionization parameter $\xi=L /\left(n_{\mathrm{e}} R^{2}\right)$, where $L$ is the luminosity and $R$ is the distance from a generic source of radiation (e.g., the hot corona) to the warm corona. For a thin disk, $z / R=$ constant $\sim 0.1$, and using Equation (1),

$$
\xi=10^{-1} \frac{\sigma_{\mathrm{T}} L}{\tau_{\mathrm{T}} R}
$$

or

$$
\xi\left(\mathrm{erg} \mathrm{cm} \mathrm{s}^{-1}\right) \sim 10^{7}\left(L / L_{\mathrm{Edd}}\right)\left(R / R_{g}\right)^{-1}
$$

where $L_{\text {Edd }}=1.26 \times 10^{46}\left(M_{8} / M_{\odot}\right) \mathrm{erg} \mathrm{s}^{-1}$ is the Eddington luminosity. So, for $L / L_{\text {Edd }}=0.1$ and $R=10 R_{g}, \xi \sim 10^{5}$ $\mathrm{erg} \mathrm{cm} \mathrm{s}^{-1}$. A different estimate can be made if the ionization is assumed to be due to the thermal emission from the accretion disk. Using the definition of the ionization parameter 

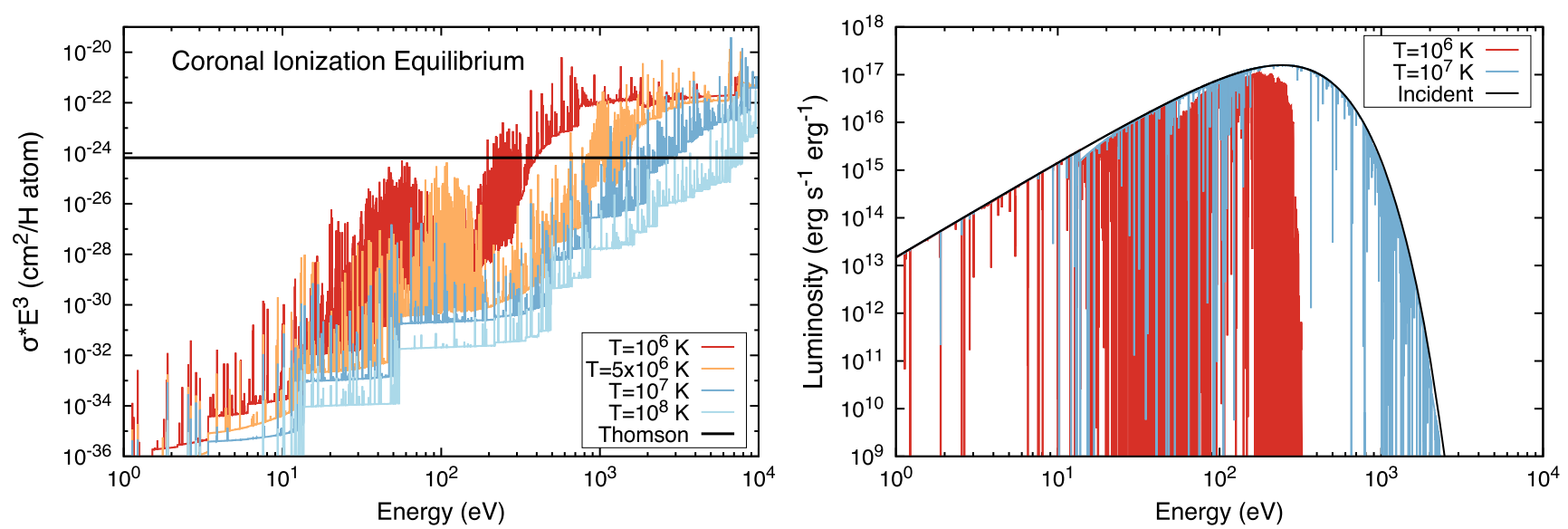

Figure 5. Calculations for a gas under CIE for different temperatures (as indicated) and the parameters that describe a warm corona: $n_{\mathrm{e}}=10^{12} \mathrm{~cm}^{-3}, \tau_{\mathrm{T}}=6.65$, and $L=10^{46} \mathrm{erg} \mathrm{s}^{-1}$. The left panel shows the photoelectric opacity as a function of energy compared to the Thomson electron opacity. The right panel shows the resulting transmitted spectra. For clarity, only two cases are displayed, those for the lowest and highest temperatures derived from fitting the warm corona model to observational data. The incident blackbody spectrum for $T=10^{7} \mathrm{~K}$ is also shown.
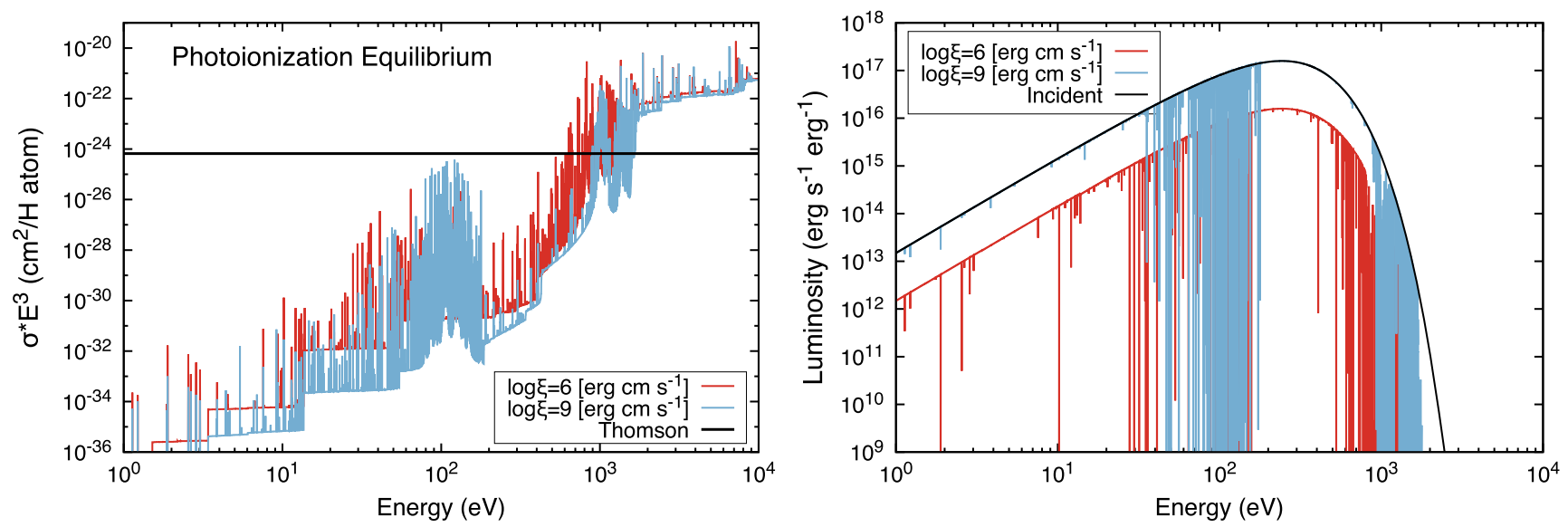

Figure 6. Calculations similar to those in Figure 5 but for the case of a gas under PIE at two different ionizations: $\xi=10^{6}$ and $10^{9}$ erg $\mathrm{cm} \mathrm{s}{ }^{-1}$. As before, the left panel shows the photoelectric opacity as a function of energy together with the Thomson opacity, while the right panel shows the resulting transmitted spectra. Even at the highest ionization predicted by our estimates of the warm corona, the original blackbody spectrum is severely modified by photoelectric absorption.

$\xi=4 \pi F / n_{\mathrm{e}}$ and the local flux from the disk,

$$
F_{d}=\frac{3 \pi}{8} \frac{G M \dot{M}}{R^{3}}
$$

and $\dot{M}=L /\left(\eta c^{2}\right)$, we find

$$
\xi=\frac{0.15 \pi^{2} \sigma_{\mathrm{T}} G}{\tau_{\mathrm{T}} \eta c^{2}} \frac{M L}{R^{2}},
$$

where $\eta \approx 0.1$ is the accretion efficiency. This last equation can be rewritten as

$$
\xi\left(\mathrm{erg} \mathrm{cm} \mathrm{s}^{-1}\right) \sim 10^{9}\left(L / L_{\mathrm{Edd}}\right)\left(R / R_{g}\right)^{-2}
$$

thus, for $L / L_{\mathrm{Edd}}=0.1$ at $R=10 R_{g}$, we get $\xi \sim 10^{6}$ $\mathrm{erg} \mathrm{cm} \mathrm{s}^{-1}$. While this expression results in a larger ionization than the estimate in Equation (4), it decreases quadratically (rather than linearly) with radius. It is also interesting that both expressions are independent of the black hole mass.

Although Equations (4) and (7) predict fairly large ionization for the warm corona, this is only true for the case of an optically thin slab. For large optical depths $\left(\tau_{T} \simeq 1\right)$, the ionization will quickly decrease in the deeper regions of the gas, and photoelectric absorption can be as or more important than the Thomson opacity. These results are generally in line with the seminal calculations presented by Ross et al. (1978), where they considered the photoionization of isothermal spheres at $T=10^{6}-10^{7} \mathrm{~K}$ with $\tau_{\mathrm{T}}=6$ and $n_{\mathrm{e}}=10^{16} \mathrm{~cm}^{-3}$. They found that despite the very high ionization at the center of the cloud, in the outer parts, ions such as Fe XXII were still dominant, producing distinct spectral features.

As before, we use the XSTAR code to test this scenario by producing the solution for a plasma under PIE using the estimates shown above, i.e., $\xi \sim 10^{6} \mathrm{erg} \mathrm{cm} \mathrm{s}^{-1}, n_{\mathrm{e}}=10^{12}$ $\mathrm{cm}^{-3}$, and $N_{\mathrm{H}}=10^{25} \mathrm{~cm}^{2}$. Using a blackbody with $k T=0.1 \mathrm{keV}$ as the input spectrum, the resulting gas temperature is $T \sim 10^{6} \mathrm{~K}$. Despite the large ionization, the photoelectric opacity near $1 \mathrm{keV}$ is still dominant over (or at least comparable to) the Thompson opacity (left panel in Figure 6). We repeated this calculation by raising the ionization to the largest value predicted by Equation (7) (i.e., $\xi \sim 10^{9}$ erg $\mathrm{cm} \mathrm{s}^{-1}$ ), but the net effect is small in reducing the photoelectric opacity. Just as in the case of CIE, the transmitted spectra show strong absorption features in the observable bandpass (right panel in Figure 6). Despite the large ionizing flux, the input spectrum is too soft to fully ionize the metals in the gas. 
None of the spectra resulting from either the CIE or PIE simulations are likely to resemble the apparently featureless broad component required to fit the soft excess. In the case of PIE, a harder spectrum extending to high energies, such as that provided by the hot corona, is likely to provide enough photons to fully ionize the medium. However, strong photoionization will raise the temperature and can only fully ionize the atmosphere if the optical depth is much smaller than that inferred from fitting the warm corona model. Moreover, strong illumination of an optically thick medium is expected to produce strong reprocessing of the photons, which is a situation that closely resembles the relativistic reflection model. This alternative scenario is discussed in the next section.

\subsection{Implications of the Relativistic Reflection Model}

The relativistic reflection model has also been proposed as a possible explanation for the soft excess in AGNs. When strong radiation is produced in the central region close the black hole, the reprocessing of the hard X-rays in the optically thick and relatively cold accretion disk is an expected consequence. If the reflection occurs close enough to the horizon, the relativistic effects will distort the spectrum, broadening and skewing all of the spectral features. Below $\sim 1 \mathrm{keV}$, a rich forest of fluorescence emission lines produced by ions with a nuclear charge lower than iron is predicted (e.g., Ross \& Fabian 2005; García \& Kallman 2010). When the gravitational blurring is extreme, these features will blend, creating a single broad and smooth excess at soft energies. When facing the difficulties in making physical sense out of a featureless and broad spectrum emitted from a warm corona, a relativistically blurred reflection spectrum provides an alternative and somewhat more consistent interpretation. However, some caveats must also be considered when adopting this model. Below, we discuss this scenario to explain the soft excess in Mrk 509.

In their analysis of a sample of 25 "bare" AGNs with Suzaku, Walton et al. (2013) fitted $~ 90 \mathrm{ks}$ XIS/PIN spectra of Mrk 509 using a model consisting of ionized and relativistic plus neutral and distant reflection components. Both components were modeled with reflionx (Ross \& Fabian 2005). A warm absorber component was also included and modeled with XSTAR. The relativistic blurring applied to the ionized reflection employed relconv (Dauser et al. 2013). Two sets of fits were performed: one with a fixed cross-normalization constant between the hard X-ray PIN and the soft X-ray XIS detectors and another in which this cross-normalization $C_{\text {PIN/XIS }}$ was allowed to vary. The uncertainty introduced by

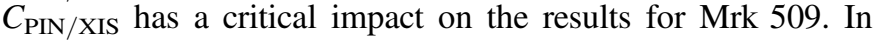
short, two vastly different pictures emerge from the fits, simply due to differences in the hard X-ray component. In the first instance of fixed $C_{\mathrm{PIN} / \mathrm{XIS}}=1.17$, the system demands a high spin, $a=0.86 \pm 0.02$, and face-on orientation (inclination of $i<18^{\circ}$ ). However, when $C_{\mathrm{PIN} / \mathrm{XIS}}$ is freed, it becomes loosely constrained $\left(C_{\mathrm{PIN} / \mathrm{XIS}}<1.06\right)$, while spin and inclination are drastically affected: $a=0.36 \pm 0.3$ and $i=50^{\circ} \pm 5^{\circ}$. This is because the hard X-ray band is essential for disentangling the power-law continuum from the ionized reflection component, which motivated the NUSTAR observations presented here.

The fit described in Section 3.2 and shown in Figure 4 demonstrates that the relativistic reflection scenario (Model 2) provides a good description of the present Suzaku and NuSTAR data for Mrk 509, with results that are broadly consistent with the high-spin fits presented by Walton et al. (2013). Moreover, our fits

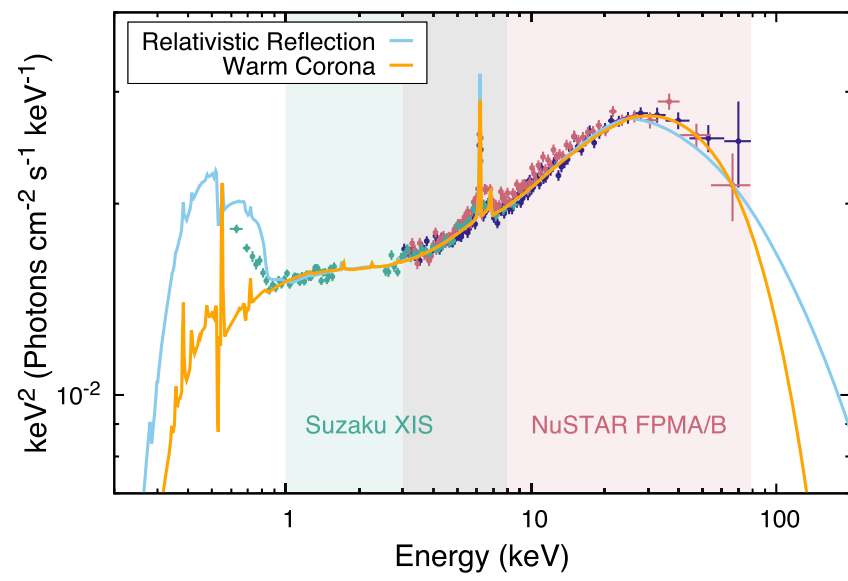

Figure 7. Unfolded spectra of Mrk 509 as seen by Suzaku XIS and NuSTAR FPMA and FPMB (data points), together with the two different scenarios for the soft excess (solid lines), the relativistic reflection (Model 2), and the warm corona (Model 1.3). The shaded regions show the data from each instrument that were included in the fits.

have been carried out with updated reflection models, which include more complete atomic data, improved radiative transfer calculations, and the possibility for higher densities in the reflector. This latter improvement is important to better describe the soft excess observed below $1 \mathrm{keV}$.

It is worth noticing the relevance of the NuSTAR data in providing high-S/N data at hard energies, where most of the reflection signatures are observed. This is particularly important because not only is our Suzaku exposure shorter than that analyzed by Walton et al. (2013), but our data lack the highenergy coverage previously provided by the PIN instrument (not longer operational in the last Suzaku cycle). In the case of the relativistic reflection Model 2, fitting the Suzaku data alone yields poor constraints to important parameters such as spin $\left(a_{*}>0.702\right)$, coronal height $\left(h=2.01_{-2.74}^{+0.29} R_{\mathrm{Hor}}\right)$, and inclination $\left(i=51^{\circ} .4 \pm 8^{\circ}\right)$. Unsurprisingly, the disk density is determined with a similar uncertainty $\left(\log n_{\mathrm{e}} / \mathrm{cm}^{-3}>18\right)$, as this parameter is mostly sensitive to the soft-energy data.

When applied to both the Suzaku and NuSTAR data, the goodness of fit for the relativistic reflection model $\left(\chi_{\nu}^{2}=1.054\right)$ is very similar to that from the fits with the warm corona picture $\left(\chi_{\nu}^{2}=1.049\right.$; Model 1.3). The similarity between the warm corona and the relativistic reflection model has also been previously discussed by Boissay et al. (2014). In Figure 7, we show these two models overplotted with the observed data. It is clear that the two models are almost identical in the energy band considered for the fits $(1-79 \mathrm{keV})$, which is shown with the shaded regions. We emphasize that data below $1 \mathrm{keV}$ were excluded given concerns in the calibration of Suzaku's instruments in this band toward the end the mission (see Section 2.3). We note, however, that when these data are included (without refitting), they seem to favor the trend predicted by the relativistic reflection model. Nevertheless, the lack of reliable data below $1 \mathrm{keV}$ limits the analysis of the present study, as we cannot fully constrain the overall shape of the soft excess. Thus, future observations with sensitive coverage of both the soft and hard energy bands will become crucial to further understanding the nature of the soft excess in Mrk 509 and several other AGNs.

The small differences between the two models seen at high energies ( $\sim 30-50 \mathrm{keV}$; Figure 7$)$ are likely due to the fact that 
the reflection models used here were calculated with a cutoff energy fixed at $300 \mathrm{keV}$, while in the warm corona fit, this parameter is allowed to vary freely. This suggests that a lower coronal temperature would be possible with the reflection model, but it is probably not very well constrained, as it does not seem to affect the fit statistics significantly.

The relativistic reflection model requires a large value for the black hole spin (consistent with its maximum value, $a_{*}$ $>0.993)$ and low coronal height $\left(h=1.53_{-0.25}^{+0.01} R_{\text {Hor }}\right)$. While high spins and compact coronae are commonly reported for AGNs, a corona placed so close to the black hole implies a very extreme configuration in which most of the radiation is focused toward the disk due to the strong light bending (Dauser et al. 2016). This configuration predicts a reflection-dominated spectrum, different from the fit achieved with Model 2 (Figure 4, right). Nonetheless, modeling the primary source of X-rays as a point source in the rotational axis is a rather simple and idealized description; thus, the derived parameters need to be interpreted with care.

The iron abundance is found to be close to its solar value $\left(A_{\mathrm{Fe}}<0.82\right)$. Fixing $A_{\mathrm{Fe}}=1$ worsens the fit by $\Delta \chi^{2} \sim 12$, having no obvious effect on the rest of the model parameters. While solar abundances are the canonical expectation, much larger $\mathrm{Fe}$ abundances are commonly derived from reflection modeling (García et al. 2018). However, recent studies indicate that high-density reflection models (like the ones used here) lead to abundances closer to solar (e.g.; Tomsick et al. 2018; Jiang et al. 2018), which is consistent with our findings. Moreover, visual inspection of the residuals reveals no obvious signs of iron emission lines after the distant reflection is accounted for (e.g., see Figure 3), suggesting that the reflection spectrum is primarily constrained by fitting the soft excess.

The large density of the accretion disk derived from our fits $\left(\log n_{\mathrm{e}} / \mathrm{cm}^{-3}>18.2\right)$ also places this source in a somewhat extreme configuration. For instance, Svensson \& Zdziarski (1994) derived analytic expressions for a hot corona around a cold $\alpha$-disk system. Using their expression for the disk density in the radiation pressure-dominated case (i.e., their Equation (8)),

$$
n_{\mathrm{e}}=\frac{1}{\sigma_{\mathrm{T}} R_{\mathrm{S}}} \frac{256 \sqrt{2}}{27} \alpha^{-1} r^{3 / 2} \dot{m}^{-2}[1-(3 / r)]^{-1}(1-f)^{-3},
$$

where $\alpha \approx 0.1$ is the standard Shakura \& Sunyaev (1973) dimensionless parameter connecting the viscosity with the gas pressure, $r=10$ is the radius in units of $R_{\mathrm{S}}=2 R_{g}, \dot{m}=0.1$, $n_{\mathrm{e}}=10^{19} \mathrm{~cm}^{-3}$, and $f$ is the fraction of the total accretion power dissipated by the corona. We find $f=0.86$, which means that most of the accretion power needs to be dissipated in the hot corona. We note that more conservative values can be found in the literature. For example, Vasudevan \& Fabian (2007) reported $f \sim 0.11-0.45$ for a sample of 54 AGNs. Nevertheless, our estimate for Mrk 509, albeit extreme, is allowed within the applicability regime of the hot corona and cold disk model.

Meanwhile, high-density reflection models like the one used here have recently been used to successfully describe the spectrum of the AGNs IRAS 13224-3809 (Parker et al. 2017; Jiang et al. 2018) and Mrk 1044 (Mallick et al. 2018), as well as the black hole binary Cyg X-1 (Tomsick et al. 2018). In all of these cases, fitting the observed soft excess results in a lower (and more physical) iron abundance in the reflector (see also discussion in Parker et al. 2018). However, in the case of
Ark 120, Porquet et al. (2018) found that the warm corona model provides a better description of the data over the relativistic reflection picture, even when high-density models were tested. In a multi-epoch study of Mrk 335, Keek \& Ballantyne (2016) showed that after fitting a reflection model above $3 \mathrm{keV}$, a constant soft excess appears to remain that is constant to the flux of the source. However, they only used standard relativistic reflection, as high-density reflection models like the ones used here were not available at the time.

One argument against the relativistic reflection scenario (and, consequently, in favor of the warm corona picture), on the other hand, is the apparent discrepancy in the correlation between the strengths of the reflection $\left(R_{\text {ref }}\right)$ and the soft excess $\left(R_{\mathrm{SE}}\right)$ components predicted by relativistic reflection models and that observed in Seyfert AGNs. Boissay et al. (2016) showed that while simulations with reflection models predict a positive correlation between $R_{\text {ref }}$ and $R_{\mathrm{SE}}$ (see also Vasudevan et al. 2014), observations of a sample of 42 AGNs show a negative correlation. They argued that this discrepancy can be overcome if the soft excess is instead modeled with warm Comptonization models. However, their sample includes data that are not simultaneous, which is likely to bias their results for sources with strong variability. More importantly, their fits implement very simplistic models for reflection, which are fundamentally incorrect to properly describe the combination of distant (nonrelativistic) and local (relativistic) reflection. In many unobscured AGNs, the narrow (unblurred) reflection component dominates the relativistic reflection signal (e.g., Ricci et al. 2014). Thus, the reflection fraction measured by Boissay et al. (2016) is likely biased toward the strength of the distant reflector. In this case, the anticorrelation with the strength of the soft excess can be simply explained by geometrical effects. For sources that are more obscured, the emission from the innermost regions will tend to be reduced, which reduces the direct continuum (increasing $R_{\text {ref }}$ ) and the local relativistic reflection component (decreasing $R_{\mathrm{SE}}$ ).

\section{Conclusions}

We have presented an analysis of the X-ray spectrum (1-79 keV) of the bright Seyfert 1 AGN Mrk 509. These data, obtained during 2015 April-June with Suzaku and NuSTAR, reveal signatures of $\mathrm{X}$-ray reprocessing from optically thick and relatively cold material, a power-law continuum, and a strong soft excess. By performing fits of different modern models, we have shown that these data can be described by a hot corona that produces the power-law continuum (modeled with a standard Comptonization model) and a distant reflection from cold material (which can be described with a variety of reflection models). Meanwhile, the soft excess can be fitted with either a warm Comptonizing corona or a relativistically blurred high-density reflection model. These two prescriptions imply two very different interpretations of the observed spectrum, and they cannot be easily distinguished on statistical grounds alone. Although the Suzaku data below $1 \mathrm{keV}$ seem to favor the relativistic reflection scenario, this energy range was excluded from the fit due to concerns regarding the quality of the instrumental calibration.

Since no model can be preferred based on the fit statistics, we have discussed in detail the physical implications of these two models. In particular, we find that the quantities required to fit the soft excess with the warm corona model-i.e., low temperature $(k T \sim 0.5-1 \mathrm{keV})$ and large optical depth 
$\left(\tau_{\mathrm{T}} \sim 10-20\right)$ - are incompatible with the physical concept of a corona, in which electron scattering is expected to be the dominant source of opacity. Using simple estimates of density, flux, and ionization parameter, we have carried out calculations of plasmas in coronal and PIE. In both cases, we found that atomic opacities will dominate over Thomson opacities, predicting very strong absorption features in the observed spectrum. Taking these simulations to the most extreme cases, we find that it is very unlikely that a warm corona can produce the soft, featureless emission required to fit the data.

On the other hand, the relativistic reflection model appears more reasonable on physical grounds. Signatures of X-ray reflection have been shown to be almost ubiquitous in most Seyfert AGN spectra, and thus it is also expected to be present in Mrk 509. The relativistic reflection model, however, requires extreme values for the spin and coronal compactness, as well as a very large density for the reflector. Although large densities are somewhat unexpected in accretion disks around supermassive black holes, we cannot discard this possibility. Therefore, based on the analysis presented here, we favor the high-density relativistic reflection scenario to explain the soft excess in Mrk 509.

Nonetheless, the present discussion is not entirely conclusive. The calculations described above do not include photon redistribution due to Comptonization in the medium or any other source of turbulent motions capable of broadening and smearing the absorption lines present in the spectra. Evidently, these effects are only relevant for the simulations at the highest temperatures $\left(T \sim 10^{7} \mathrm{~K}\right)$. For lower temperatures, the drastic modification of the spectrum due to the strong absorption prevents this model from reproducing the soft excess. Detailed radiative transfer calculations covering larger optical depths, Comptonization, velocity components, and the effects of the response of current instruments are necessary to fully explore this problem. Such calculations are well outside the scope of the present work and thus will be featured in a future publication.

Finally, deeper observations of this source should be able to confirm or deny the presence of relativistic reflection. To clearly distinguish between the narrow and broad components, future missions flying microcalorimeters, such as XRISM (Tashiro et al. 2018), Athena (Nandra et al. 2013), and Lynx (Özel 2018), will become crucial. However, in order to detect the shift of the Compton hump between the relativistic and nonrelativistic reflection, the focusing of hard photons with a larger effective area than $N U S T A R$ is necessary. The concept mission HEX-P (Madsen et al. 2018) will offer these capabilities. Likewise, observations with instruments with broadband coverage and good sensitivity to both soft and hard energy bands, such as STROBE-X (Ray et al. 2018), will help to break model degeneracies and further understand the nature of the soft excess in Mrk 509 and many other AGNs.

We thank P.O. Petrucci, J. Malzac, B. Czerny, A. Różańska, C. Done, and the members of the FERO collaboration for insightful discussions that promoted many aspects of this paper. We also thank F. Ursini for comments that improved the manuscript.

J.A.G. acknowledges support from NASA grant NNX15AV31G and the Alexander von Humboldt Foundation. R.M.T.C. has been supported by NASA grant 80NSSC177K0515. M.B. acknowledges support from the black hole initiative at Harvard University, which is funded by a grant from the John Templeton Foundation. J.F.S. has been supported by NASA Einstein Fellowship grant PF5160144. F.T. acknowledges support by the Programma per Giovani Ricercatori-anno 2014 "Rita Levi Montalcini." L.L. acknowledges support from NASA through grant No. NNX15AP24G C.R. acknowledges support from the CONICYT+PAI Convocatoria Nacional subvención a instalación en la academia convocatoria año 2017 PAI77170080.

This work was partially supported under NASA contract No. NNG08FD60C and made use of data from the NuSTAR mission, a project led by the California Institute of Technology, managed by the Jet Propulsion Laboratory, and funded by the National Aeronautics and Space Administration. We thank the NUSTAR Operations, Software, and Calibration teams for support with the execution and analysis of these observations. This research has made use of the NuSTAR Data Analysis Software (NuSTARDAS), jointly developed by the ASI Science Data Center (ASDC, Italy) and the California Institute of Technology (USA).

Facilities: NuSTAR, Suzaku (XIS).

Software: XSPEC (v12.9.0d; Arnaud 1996), MYTORUS (Murphy \& Yaqoob 2009), BORUS02 (Baloković et al. 2018), XILLVER (García \& Kallman 2010; García et al. 2013), RELXILL (v1.2.0; García et al. 2014; Dauser et al. 2014), XSTAR (v2.41; Kallman \& Bautista 2001), NUSTARDAS (v1.6.0).

\section{ORCID iDs}

Javier A. García (1) https://orcid.org/0000-0003-3828-2448 Erin Kara (10 https://orcid.org/0000-0003-0172-0854 Dominic Walton (i) https://orcid.org/0000-0001-5819-3552 Thomas Dauser (iD https://orcid.org/0000-0003-4583-9048 Efrain Gatuzz (ib https://orcid.org/0000-0002-3252-9633 Mislav Balokovic (iD https://orcid.org/0000-0003-0476-6647 James F. Steiner (iD https://orcid.org/0000-0002-5872-6061 Francesco Tombesi (iD https://orcid.org/0000-0002-6562-8654 Riley M. T. Connors (iD https://orcid.org/0000-0002-8908-759X Timothy R. Kallman (iD https://orcid.org/0000-0002-5779-6906 Fiona A. Harrison (D) https://orcid.org/0000-0003-2992-8024 Andrew Fabian (1) https://orcid.org/0000-0002-9378-4072 Jörn Wilms (10) https://orcid.org/0000-0003-2065-5410 Daniel Stern (i) https://orcid.org/0000-0003-2686-9241 Lauranne Lanz (i) https://orcid.org/0000-0002-3249-8224 Claudio Ricci (1) https://orcid.org/0000-0001-5231-2645 David R. Ballantyne (i) https://orcid.org/0000-00018128-6976

\section{References}

Arnaud, K. A. 1996, in ASP Conf. Ser.101, Astronomical Data Analysis Software and Systems V, ed. G. H. Jacoby \& J. Barnes (San Francisco, CA: ASP), 17

Arnaud, K. A., Branduardi-Raymont, G., Culhane, J. L., et al. 1985, MNRAS, 217, 105

Baloković, M., Brightman, M., Harrison, F. A., et al. 2018, ApJ, 854, 42 Baloković, M., Matt, G., Harrison, F. A., et al. 2015, ApJ, 800, 62 Boissay, R., Paltani, S., Ponti, G., et al. 2014, A\&A, 567, A44 Boissay, R., Ricci, C., \& Paltani, S. 2016, A\&A, 588, A70 Brenneman, L. W., Madejski, G., Fuerst, F., et al. 2014, ApJ, 788, 61 Brightman, M., \& Nandra, K. 2011, MNRAS, 413, 1206 Cappi, M., Tombesi, F., Bianchi, S., et al. 2009, A\&A, 504, 401 Costantini, E., Kriss, G., Kaastra, J. S., et al. 2016, A\&A, 595, A106 Crummy, J., Fabian, A. C., Gallo, L., \& Ross, R. R. 2006, MNRAS, 365, 1067 Czerny, B., \& Elvis, M. 1987, ApJ, 321, 305

Czerny, B., Nikołajuk, M., Różańska, A., et al. 2003, A\&A, 412, 317 
Dauser, T., García, J., Parker, M., Fabian, A., \& Wimls, J. 2014, MNRAS, 444, 100

Dauser, T., García, J., Walton, D. J., et al. 2016, A\&A, 590, A76

Dauser, T., Garcia, J., Wilms, J., et al. 2013, MNRAS, 430, 1694

Done, C., Davis, S. W., Jin, C., Blaes, O., \& Ward, M. 2012, MNRAS, 420, 1848

Fabian, A. C., Ballantyne, D. R., Merloni, A., et al. 2002, MNRAS, 331, L35

Fabian, A. C., Zoghbi, A., Ross, R. R., et al. 2009, Natur, 459, 540

Fisher, K. B., Huchra, J. P., Strauss, M. A., et al. 1995, ApJS, 100, 69

García, J., Dauser, T., Lohfink, A., et al. 2014, ApJ, 782, 76

García, J., Dauser, T., Reynolds, C. S., et al. 2013, ApJ, 768, 146

García, J., \& Kallman, T. R. 2010, ApJ, 718, 695

García, J. A., Dauser, T., Steiner, J. F., et al. 2015, ApJL, 808, L37

García, J. A., Fabian, A. C., Kallman, T. R., et al. 2016, MNRAS, 462, 751

García, J. A., Kallman, T. R., Bautista, M., et al. 2018, in ASP Conf. Ser. 515,

Workshop on Astrophysical Opacities, ed. C. Mendoza, S. Turck-Chieze, \& J. Colgan (San Francisco, CA: ASP), 282

George, I. M., \& Fabian, A. C. 1991, MNRAS, 249, 352

Gierliński, M., \& Done, C. 2004, MNRAS, 349, L7

Haardt, F. 1993, ApJ, 413, 680

Harrison, F. A., Craig, W. W., Christensen, F. E., et al. 2013, ApJ, 770, 103

Jiang, J., Parker, M. L., Fabian, A. C., et al. 2018, MNRAS, 477, 3711

Jin, C., Done, C., Ward, M., Gierliński, M., \& Mullaney, J. 2009, MNRAS, 398, L16

Kaastra, J. S., Ebrero, J., Arav, N., et al. 2014, A\&A, 570, A73

Kaastra, J. S., Petrucci, P.-O., Cappi, M., et al. 2011, A\&A, 534, A36

Kalberla, P. M. W., Burton, W. B., Hartmann, D., et al. 2005, A\&A, 440, 775

Kallman, T., \& Bautista, M. 2001, ApJS, 133, 221

Kara, E., García, J. A., Lohfink, A., et al. 2017, MNRAS, 468, 3489

Kaufman, J., Blaes, O. M., \& Hirose, S. 2018, MNRAS, 476, 5548

Keck, M. L., Brenneman, L. W., Ballantyne, D. R., et al. 2015, ApJ, 806, 149

Keek, L., \& Ballantyne, D. R. 2016, MNRAS, 456, 2722

Kettula, K., Nevalainen, J., \& Miller, E. D. 2013, A\&A, 552, A47

Koyama, K., Tsunemi, H., Dotani, T., et al. 2007, PASJ, 59, 23

Krolik, J. H., \& Kallman, T. R. 1984, ApJ, 286, 366

Laor, A. 1991, ApJ, 376, 90

Leighly, K. M. 1999, ApJS, 125, 317

Liu, Y., \& Li, X. 2015, MNRAS, 448, L53

Madsen, K. K., Beardmore, A. P., Forster, K., et al. 2017, AJ, 153, 2

Madsen, K. K., Harrison, F., Broadway, D., et al. 2018, Proc. SPIE, 10699, $106996 \mathrm{M}$

Madsen, K. K., Harrison, F. A., Markwardt, C. B., et al. 2015, ApJS, 220, 8

Magdziarz, P., Blaes, O. M., Zdziarski, A. A., Johnson, W. N., \& Smith, D. A. 1998, MNRAS, 301, 179

Mallick, L., Alston, W. N., Parker, M. L., et al. 2018, MNRAS, 479, 615

Marinucci, A., Bianchi, S., Braito, V., et al. 2018, MNRAS, 478, 5638

Marinucci, A., Tortosa, A. \& NuSTAR AGN Physics Working Group 2016, AN, 337, 490

Markoff, S., Nowak, M. A., \& Wilms, J. 2005, ApJ, 635, 1203

Matt, G., Perola, G. C., \& Piro, L. 1991, A\&A, 247, 25

Matt, G., Perola, G. C., Piro, L., \& Stella, L. 1992, A\&A, 257, 63

Mehdipour, M., Branduardi-Raymont, G., Kaastra, J. S., et al. 2011, A\&A, 534, A39

Middleton, M., Done, C., Ward, M., Gierliński, M., \& Schurch, N. 2009, MNRAS, 394, 250

Miniutti, G., Ponti, G., Greene, J. E., et al. 2009, MNRAS, 394, 443
Morini, M., Lipani, N. A., \& Molteni, D. 1987, ApJ, 317, 145

Murphy, K. D., \& Yaqoob, T. 2009, MNRAS, 397, 1549

Nandra, K., Barret, D., Barcons, X., et al. 2013, arXiv:1306.2307

Nardini, E., Fabian, A. C., \& Walton, D. J. 2012, MNRAS, 423, 3299

Nardini, E., Porquet, D., Reeves, J. N., et al. 2016, ApJ, 832, 45

Özel, F. 2018, NatAs, 2, 608

Parker, M. L., Miller, J. M., \& Fabian, A. C. 2018, MNRAS, 474, 1538

Parker, M. L., Pinto, C., Fabian, A. C., et al. 2017, Natur, 543, 83

Peterson, B. M., Ferrarese, L., Gilbert, K. M., et al. 2004, ApJ, 613, 682

Petrucci, P. O., Merloni, A., Fabian, A., Haardt, F., \& Gallo, E. 2001, MNRAS, 328, 501

Petrucci, P.-O., Paltani, S., Malzac, J., et al. 2013, A\&A, 549, A73

Petrucci, P.-O., Ursini, F., De Rosa, A., et al. 2018, A\&A, 611, A59

Piconcelli, E., Jimenez-Bailón, E., Guainazzi, M., et al. 2005, A\&A, 432, 15

Ponti, G., Cappi, M., Costantini, E., et al. 2013, A\&A, 549, A72

Porquet, D., Reeves, J. N., Matt, G., et al. 2018, A\&A, 609, A42

Porquet, D., Reeves, J. N., O’Brien, P., \& Brinkmann, W. 2004, A\&A, 422, 85

Pounds, K. A., Nandra, K., Fink, H. H., \& Makino, F. 1994, MNRAS, 267, 193

Pounds, K. A., Warwick, R. S., Culhane, J. L., \& de Korte, P. A. J. 1986, MNRAS, 218, 685

Ray, P. S., Arzoumanian, Z., Brandt, S., et al. 2018, Proc. SPIE, 10699, 1069919

Ricci, C., Trakhtenbrot, B., Koss, M. J., et al. 2017, ApJS, 233, 17

Ricci, C., Ueda, Y., Paltani, S., et al. 2014, MNRAS, 441, 3622

Risaliti, G., Harrison, F. A., Madsen, K. K., et al. 2013, Natur, 494, 449

Różańska, A., Malzac, J., Belmont, R., Czerny, B., \& Petrucci, P.-O. 2015, A\&A, 580, A77

Ross, R. R., \& Fabian, A. C. 2005, MNRAS, 358, 211

Ross, R. R., Weaver, R., \& McCray, R. 1978, ApJ, 219, 292

Shakura, N. I., \& Sunyaev, R. A. 1973, A\&A, 24, 337

Singh, K. P., Garmire, G. P., \& Nousek, J. 1985, ApJ, 297, 633

Smith, R. A. N., Page, M. J., \& Branduardi-Raymont, G. 2007, A\&A, 461, 135

Svensson, R., \& Zdziarski, A. A. 1994, ApJ, 436, 599

Tashiro, M., Maejima, H., Toda, K., et al. 2018, Proc. SPIE, 10699, 1069922

Tomsick, J. A., Parker, M. L., García, J. A., et al. 2018, ApJ, 855, 3

Tortosa, A., Bianchi, S., Marinucci, A., Matt, G., \& Petrucci, P. O. 2018 A\&A, 614, A37

Tortosa, A., Marinucci, A., Matt, G., et al. 2017, MNRAS, 466, 4193

Ursini, F., Boissay, R., Petrucci, P.-O., et al. 2015, A\&A, 577, A38

Vasudevan, R. V., \& Fabian, A. C. 2007, MNRAS, 381, 1235

Vasudevan, R. V., Mushotzky, R. F., Reynolds, C. S., et al. 2014, ApJ, 785, 30

Vaughan, S., Edelson, R., Warwick, R. S., \& Uttley, P. 2003, MNRAS, 345,1271

Verner, D. A., Ferland, G. J., Korista, K. T., \& Yakovlev, D. G. 1996, ApJ, 465,487

Walter, R., \& Fink, H. H. 1993, A\&A, 274, 105

Walton, D. J., Nardini, E., Fabian, A. C., Gallo, L. C., \& Reis, R. C. 2013, MNRAS, 428, 2901

Walton, D. J., Risaliti, G., Harrison, F. A., et al. 2014, ApJ, 788, 76

Walton, D. J., Tomsick, J. A., Madsen, K. K., et al. 2016, ApJ, 826, 87

Wilms, J., Allen, A., \& McCray, R. 2000, ApJ, 542, 914

Woo, J.-H., \& Urry, C. M. 2002, ApJ, 579, 530

Xu, Y., Baloković, M., Walton, D. J., et al. 2017, ApJ, 837, 21

Yaqoob, T., Murphy, K. D., Griffiths, R. E., et al. 2007, PASJ, 59, 283

Zdziarski, A. A., Johnson, W. N., \& Magdziarz, P. 1996, MNRAS, 283, 193

Życki, P. T., Done, C., \& Smith, D. A. 1999, MNRAS, 309, 561 